\title{
Regards Sur Les Impacts Environnementaux De Projet d'Exploitation Minière Semi - Industrielle Dans Le Bassin Du Congo: Cas De La Rivière Aruwimi (Territoire De Basoko, RD Congo)
}

\author{
Mangambu Mokoso Jean De Dieu \\ Professeur, Laboratoire de Systématique Végétale, Biodiversité \& \\ Management des Écosystèmes (LSVBME), Département de Biologie, \\ Faculté des Sciences, Université Officielle de Bukavu, RDC \\ Asimbo Bondoo Norbert \\ Assistant et Master de Spécialisation en Développement, Environnement et \\ Sociétés, Département de l'Economie Publique, Faculté des Sciences \\ Economiques, Université de Kisangani, RDC

\section{Ekele Mbenga Robert} \\ Professeur, Département des Gestions des Entreprises, Entrepreneuriat et \\ Organisation du travail, Faculté de Psychologie et des Sciences de \\ l'Education, Université Pédagogique Nationale, Kinshasa, RDC
}

\section{Doi:10.19044/esj.2021.v17n29p328}

Submitted: 14 June 2021

Accepted: 20 August 2021

Published: 31 August 2021
Copyright 2021 Author(s)

Under Creative Commons BY-NC-ND 4.0 OPEN ACCESS

Cite As:

Mangambu Mokoso J.D., Asimbo Bondoo N. \& Ekele Mbenga R. (2021). Regards Sur Les Impacts Environnementaux De Projet d'Exploitation Minière Semi - Industrielle Dans Le Bassin Du Congo : Cas De La Rivière Aruwimi (Territoire De Basoko, RD Congo). European Scientific Journal, ESJ, 17(29), 328.

https://doi.org/10.19044/esj.2021.v17n29p328

\section{Résumé}

Le présent article se base sur l'étude d'impacts environnementaux du projet de l'exploitation minière semi - industrielle le long de la rivière Aruwimi autour de la cité de Liambe et ses environs en Territoire de Basoko par la société chinoise Xiang Jiang Mining Sarl. Une enquête quantitative et des cas qualitatifs ont été menés sur cinq couches différentes de la population du milieu. Après les analyses sur les conséquences de cette exploitation qu'envisage la société chinoise, notre étude a démontré que 76,63\% de la population enquêtée ne sont ni formées et ni sensibilisées sur le nouveau code minier modifié en 2018 par la RD Congo et 79,5 \% ne maitrisent ni la 
nouvelle loi et ni leur droit. Les discussions menées auprès de ces peuples nous indiquent que cette tentative d'exploitation est caractérisée par un manque de formalisme et d'opacité. Actuellement, ce cours d'eau apparaît comme des réceptacles importants de tensions et conflits, en raison des abus majeurs qui se sont observés durant l'exploitation minière dans le territoire de Banalia. Ils considèrent cette tentative d'exploitation comme illicite qui est associée à nombreux phénomènes négatifs, tels que des conflits fonciers, des violations des droits de l'homme, la perturbation de l'écosystème aquatique et de procédure cavalière au mépris du processus réglementaire et légal. Face à ce danger, nous avons décidé de lancer ce cri d’alarme pour alerter la société sur le désastre écologique, environnemental et socio-économique qui guette les populations riveraines d'Aruwimi et exprimer ainsi notre désapprobation sur cette exploitation minière.

Mots clés : Exploitation Minière, Projet d'Atteinte Environnementale, Rivière Aruwimi, Territoire De Basoko, RDC

\title{
Insights Into The Environmental Impacts Of Semi-Industrial Mining Projects In The Congo Basin: Case Of The Aruwimi River (Basoko Territory In DR Congo)
}

\section{Mangambu Mokoso Jean De Dieu}

Professor, Laboratory of Plant Systematics, Biodiversity and Ecosystem Management (LSVBME), Department of Biology, Faculty of Science, Official University, DRC

Asimbo Bondoo Norbert

Assistant and Specialized Master in Development, Environment and Societies, Department of Public Economy, Faculty of Economics, University of Kisangani, DRC

\section{Ekele Mbenga Robert}

Professor, Department of Business Management, Entrepreneurship and Work Organization, Faculty of Psychology and Educational Sciences, National Pedagogical University, Kinshasa, DRC

\begin{abstract}
This paper focuses on the environmental impact assessment of the semi-industrial mining project of the Chinese company Xiang Jiang Mining Sarl along the Aruwimi River in Basoko Territory, around the Liambe city and its surroundings. Qualitative and quantitative surveys were conducted on five different strata of the local population. After analysis of the consequences of the planned exploitation by this company, this study clearly demonstrated that
\end{abstract}


$76.63 \%$ of the population is neither trained nor aware of the new mining code as modified in 2018 by the Congolese authorities. Furthermore, $79.5 \%$ of the people are not informed about the new law and their rights. Moreover, the discussions held with the population indicate that this attempt at exploitation is informal and lacks transparency. Today, this river appears to be a more important source of tensions and conflicts due to major abuses which are often observed in the mining zone. Such exploitations generate conflicts between the population and foreign firms, and the illicit exploitation of natural resources is associated with a number of negative phenomena, such as land conflicts, violation of human rights, destruction of the environment, disruption of the aquatic ecosystem, and a rider procedure in defiance of the regulatory and legal process. Faced with this danger, warning has been given to the local population in regard to an ecological, environmental, and socio-economic disaster that awaits the people living in the vicinity of Aruwimi and to express their total disapproval.

Keywords: Environmental Threats Project, Mining, Aruwimi River, Basoko Territory, DRC

\section{Introduction}

La dégradation des eaux et des terres arables continue d'être une inquiétude mondiale, en raison de la menace qu'elle représente à la fois pour le fonctionnement des écosystèmes et le bien-être des communautés humaines. Cela est dû soit à la croissance démographique, aux progrès technologiques, à l'industrialisation, à l'expansion agricole à tous les niveaux, et ont été le moteur d'un mode de développement qui a un impact dramatique sur l'environnement naturel (Lara-Pulido et al., 2018). Ces changements globaux menacent les divers services écosystémiques rendus par ses biocénoses et les biotopes dans le Bassin du Congo qui est le deuxième poumon planétaire et joue un rôle capital sur la régulation du climat local et mondial (Bonan, 2008 ; Breshears et al., 2011). Les écosystèmes du Bassin du Congo nous produisent du bois, assurent la séquestration de carbone, la quantité et la qualité de la recharge en eau des nappes, ou la prévention de la désagrégation des terres... (Dixon et al., 1994 ; Stratos, 2011). Il faut évaluer le devenir des écosystèmes actuels et déterminer les capacités d'adaptation de ces écosystèmes face aux différents facteurs dus à l'exploitation des ressources naturelles impliquées dans les changements et les transformations relatives à l'usage des terres (Andre et al., 2010, Breshears et al., 2019).

Au regard de cette réalité, aujourd'hui, le monde est confronté à des enjeux environnementaux à tous les niveaux : micro, méso et macro. Les grands défis à relever sont majeurs à la suite de l'importance inestimable que revêt la gestion des terres, des eaux et des ressources naturelles renouvelables 
(l'eau ou la terre) et essentiellement non renouvelables (les diamants, l'or etc). Le contrôle sur ses ressources naturelles ou leur utilisation est également un facteur important de tensions, voire de conflits entre groupes sociaux (Keenan et al., 2002 ; Berzins et al., 2007). Dans le cadre d'exploitation minière, les rejets miniers peuvent impacter négativement la santé des employés et des populations des localités abritant les mines, avec des conséquences inévitables sur le bien-être général des populations riveraines (Wakenge, 2019).

Selon l'Institut de la Francophonie pour le Développement Durable (IFDD, 2019), actuellement, l'amplification de l'exploitation des ressources de la planète est à la genèse d'une dégradation de l'environnement qui peut localement bouleverser les conditions de vie des communautés, notamment dans les pays en développements. L'exploitation des ressources à grande échelle comme les mines peut alors donner lieu à des conflits où l'environnement est en quelque sorte « un champ de bataille entre les intérêts divergents » des firmes et des populations locales. L'étude des conflits environnementaux autour du projet de l'exploitation des ressources constitue une orientation relativement récente de la recherche (Homer-Dixon, 1994), à la charnière de la science de la nature d'une part, de la sociologie et de l'économie de développement d'autre part (Schröter et al., 2005; Breshears et al., 2019) pour tenter de répondre aux défis environnementaux de notre ère à la question d'utilisation et de vente de divers services écosystémiques (Hynes et al., 2018).

Dans cette optique, l'Assemblée Générale des Nations-Unies a adopté le 27 septembre 2015, les 17 Objectifs du Développement Durable déclinés en 169 cibles pour la période 2015 à 2030. Ces objectifs sont un programme commun vers un monde durable, qui vise à piloter une transition à la fois économique, sociétale et environnementale, vers un développement soutenable pour la planète et pour l'homme. Cette vision laisse entendre qu'il n’y a pas de croissance économique sans qu'il y ait une gérance durable des ressources naturelles et sans solidarité ainsi qu'à l'exclusion des progrès humains. Face à cette réalité globalisante, Breshears et al. (2019), demandent que le monde actuel travaille dans cette perspective et toutes les stipulations doivent être mises en œuvre pour la promotion du nouveau paradigme industriel et économique (économie circulaire) et pour empêcher l'écroulement de notre maison commune.

La République Démocratique du Congo (RDC) est un pays immense (le deuxième plus grand de l'Afrique), aux innombrables ressources naturelles riches et variées. En plus d'une faune et d'une flore impressionnantes, elle possède l'un des plus grands domaines de forêt équatoriale au monde et d'importantes réserves en pétrole. Son sous-sol regorge de minerais et métaux divers (Coghlan et al., 2007 ; Wakenge, 2019). L'immense fortune minière du bassin du Congo résulte de l'histoire géologique et de la position unique du 
bassin de sédimentation de la cuvette centrale. En effet, la RDC immerge dans une mer intérieure qui remplissait la cuvette centrale. Celle-ci avait recueilli en bassin de sédimentation, lors de la désertification du Sahara, des quantités incommensurables des débris organiques du Sahara (Dmoergue \& Mpoyi, 2012).

$\mathrm{Au}$ vue de toutes les convoitises qui apparaissent à la suite de la concentration dans le bassin du Congo de beaucoup des richesses naturelles, singulièrement minières, plusieurs observateurs et chercheurs estiment qu'il serait pratiquement impossible que la RDC vive en paix (Kamundala, 2012). Ils pourraient avoir raison au regard du cafouillage qui bat son plein dans les zones en conflits dont l'issue heureuse ne pointe pas encore à l'horizon. Une bonne raison pour interpeler le congolais est celui de l'exproprier de ses richesses. Dans cette perspective, les mines étant des ressources non renouvelables, au niveau de la cuvette centrale du bassin du Congo comme ceux de la rivière Aruwimi seront disparus avec une exploitation non contrôlée et anarchique (Abadie, 2008). La prise de conscience de cette gestion des richesses tend à faire l'unanimité entre les politiques et les scientifiques. Cette façon d'apercevoir le modèle économique traditionnel est en train de subir des mutations vers un système plus sociétal et durable (Coulibaly, 2013).

En RDC, plusieurs articles et ouvrages ont mis en défit l'exploitation minières par les firmes étrangers qui ne respectent pas la législation congolaise. Mazalto (2009), Kamundala (2012) et Grzybowski (2012) ont montré que l'exploitation des ressources minières industrielles apparaît comme négative à la fois pour l'environnement et le développement socioéconomique. Les ONGs et mouvement citoyens qui soutiennent la population locale dans leur combat contre les projets miniers ont trouvé une certaine légitimation auprès de l'opinion publique pour ses atteintes aux droits de l'homme qui sont liées à l'absence d'une application efficace des lois dans ce secteur.

Les études menées par le Programme des Nations Unies pour l'Environnement (PNUE) en 2016 sur base d'un travail de terrain à Butuzi dans le Sud-Kivu et à Some dans l'Ituri ont montré que le secteur minier contribue à la pollution de la nature, à la déforestation, à la perte de biodiversité et à un problème lié à la sécurité du travail de la population indigène. Les recherches effectuées par la commission Justice et Paix de Wallonie -Bruxelles (2019), et par Cyuzuzo (2020) ont montré que l'un des problèmes centraux du secteur de l'exploitation minière pour la RD Congo en est sa gestion et le non-respect du cadre législatif des textes élaborés. Malgré ces conséquences néfastes perceptibles, les activités d'orpaillage et d'exploitation minière continuent à se développer. Mais, le rôle de sensibilisation que l'Etat et les Organisations 
doivent jouer pour éveiller les consciences souffre des manquements (Lassourd, 2019).

Cette étude a pour objectif, d'analyser les conséquences environnementales du projet de l'exploitation minière dans le lit de la rivière Aruwimi en Territoire de Basoko qui se situe au centre de la cuvette centrale. Notre démarche tient compte de la contradiction qu'on voudrait entretenir entre le discours de lutte contre la pauvreté et celui du développement durable. Cette dichotomie n'est pas encore résolue en RDC, malgré la mise en place d'une norme conservationniste depuis 2002 et son renforcement en 2018. Dans cette perspective, nous cherchons à comprendre comment l'exploitation minière industrielle détériore les autres ressources naturelles, dégrade le cadre de vie, crée l'instabilité et les conflits sociaux et perturbe la paix sociale. Ces entorses sont une réalité en milieux rural congolais avec son corollaire d'insécurité socio-politique et d'impacts environnementaux négatifs.

\section{Approche Methodologique Milieu d'étude}

Cette étude est effectuée dans le Territoire de Basoko qui est une Entité Territoriale Déconcentrée (ETD) de la province de la Tshopo en RDC. D'une superficie de 22. $436 \mathrm{~km}^{2}$ (Figure 1, De Saint Moulin, 2012). Il se situe à une altitude qui varie entre 120 à $800 \mathrm{~m}$, une latitude de 1 à $2^{\circ}$ Nord et une longitude de 20 à $26^{\circ}$ Est, avec une taille de population estimée à 332.117 habitants, soit 15 habitants $/ \mathrm{Km}^{2}$. qui se trouve entièrement dans le bassin du Congo (source : Anonyme, 2016, Données du Bureau d'Etat civil de Basoko 2016).

Son climat est du type équatorial avec alternance de la saison sèche et la saison pluvieuse. La saison sèche va de mi-décembre à mi-mars, tandis que la saison pluvieuse va de mi-mars à mi-juin pour la saison culturale $\mathrm{A}$ et de mi-juin à mi-août ainsi que de mi-août à mi-décembre pour la saison culturale B. Sa température moyenne ambiante est supérieure ou égale à $25^{\circ} \mathrm{C}$. L'amplitude thermique est nulle, les précipitations sont abondantes et non uniformément reparties au cours de l'année. Les pluviométries maximales sont enregistrées au mois d'avril et d'octobre, l'humidité et la chaleur sont constantes (Asimbo \& Mangambu, 2021, sous presse). 


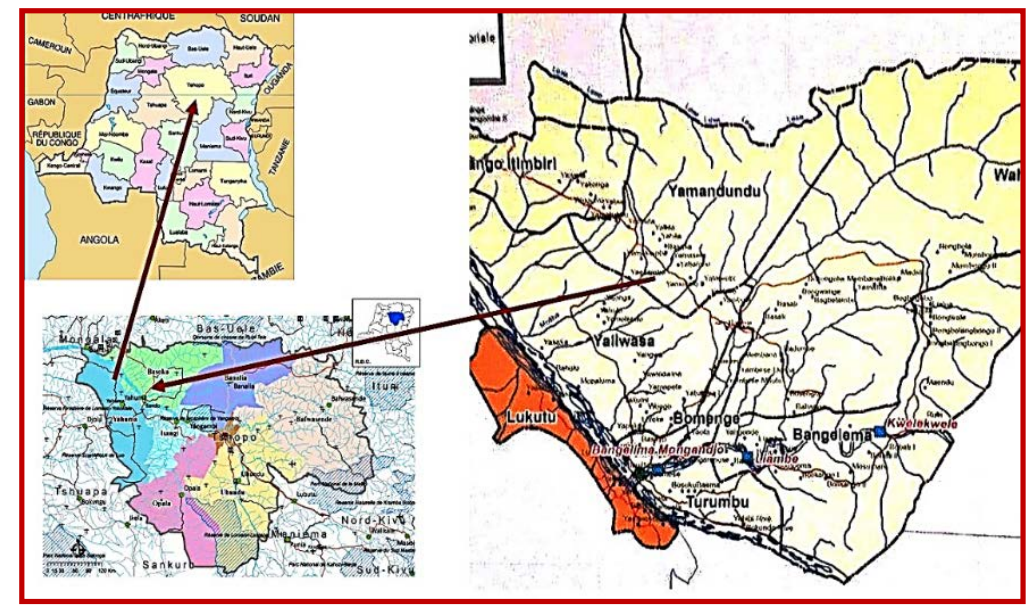

Figure 1. La carte du territoire de Basoko (De Saint Moulin, 2012)

Son relief est dominé par des grandes étendues de terrain plat de la plaine centrale orientale et avec plusieurs petites collines. Son sous-sol est riche en minerais (or, diamant, etc., Mangambu, 2021 sous presse). La végétation du territoire de Basoko est dominée par la forêt équatoriale sempervirente primaire ou secondaire, où dominent des essences exploitables telles que Pericopsis elata (Afromosia), Gilbertiodendron dewevrei (Limbalo), Milicia excelsa, Uapaca guinensis, Prioria balsamifera, Entandophragma ssp (Liboyo), etc. Les espèces phares de la faune de Basoko sont : les Hippopotames, Crocodiles, Eléphants, Singes (Chimpanzé commun, Colobes, Babouin...), Serpents (Vipères, Pitons, Mambas...), les divers oiseaux et poissons d'eaux douces (Clarias sp (Ngolo en langue locale), Auchenoglanis sp. (Foka), Hydrocynus sp. (Mbenga), Distichodus sp. (Mboto), Heterobranchus sp. (Kamba).., Mangambu, 2021 sous presse)).

La rivière Aruwimi, site d'étude est appelée aussi Lohale par la population de Basoko. Elle coule dans le Nord et le Nord-Est du pays, dans les régions de l'Ituri, des Uélés et de la Tshopo. Son cours supérieur porte le nom d'Ituri qui prend naissance dans la région de savanes située au nord du bassin de la rivière Kibali (Oberiuele, 2011).

\section{Collecte et traitement des données Collecte des données}

Les recherches se sont déroulées de janvier - juin 2019 et d'août décembre 2020 dans 22 villages et deux cités de territoire de Basoko. Pour récolter les données, nous avons utilisé trois techniques: documentaire, focus group et entretiens semi-structurés.

Pour la technique documentaire, nous avions consulté les archives du Territoire de Basoko, ceux des Divisions Provinciales des Mines et Géologie, des Ministères Nationaux de Mines et de l'Environnement...). 
Les entretiens (focus group) et les enquêtes (entretiens semistructurés) étaient orientés vers les acteurs de la société civile, les chercheurs locaux, les étudiants, les élèves des écoles secondaires, les commerçants, les pécheurs \& les agriculteurs, les autochtones, et d'autres riverains ainsi que les agents de l'Administration Territoriale.

En vue de nous rendre compte de la divergence des opinions émises par les intervenants, les interviews et les enquêtes avaient tenu compte du genre tel que proposé par Moreau et al. (2004), Touré (2010), et GavardPerret et al. (2011).

\section{Traitement des données}

Pour déterminer la taille de l'échantillon, la formule de Bouchard (2010) a été utilisée. Pour une population infinie allant jusqu’à 1000000 d'individus, on prend un échantillon de 96 personnes avec une marge d'erreur de 10 pour chaque catégorie.

$$
\mathrm{NC}=\frac{n \times N}{n+N}=\frac{96 \times 59268}{96+59268}=95,5 \text { soit } 96 \text { personnes. }
$$

NC : Taille de l'échantillon corrigé, $N=$ la taille de la population-mère (taille de l'univers) et $n=$ la taille de l'échantillon pour une population infinie ou Taille de l'échantillon pour l'univers fini.

Suivant la méthode non probabiliste Howell (2004), Acar et Sun (2013), 742 personnes ont été enquêtées avec une moyenne de 106 personnes par catégorie.

Le traitement des données a mis l'accent sur la recherche des pourcentages et des moyennes arithmétiques des données qualitatives mesurées. La mesure des liaisons a été faite en recourant au test de Khi-deux et de l'analyse de régression de valeur $p(<0,05)$. Les conditions de validité de ce dernier test ont été vérifiées avant son application (Howell, 2004). L'analyse de régression utilisée a permis la modélisation des relations entre différentes variables (dépendantes et indépendantes). Ces analyses ont été réalisées grâce à des logiciels Excel et $\mathrm{R}$.

\section{Perspective Des Impacts Environnementaux Du Projet De L'exploitation Miniere Semi - Industrielle}

Dans le contexte actuel où les idées du développement durable éprouvent une audience graduelle, le fait qu'une activité par essence même temporaire, puisse transformer profondément le cadre naturel d'une région et compromettre peut-être définitivement une autre forme d'exploitation après la fermeture de la mine, apparaît de plus en plus difficile à accepter. C'est dans cet esprit que les mouvements environnementalistes de Basoko qui se réclament pour la plupart de l'altermondialisme combattent l'industrie minière 
dans laquelle ils voient une des formes les plus scandaleuses de l'exploitation du Sud par le Nord et même l'une des causes de l'absence de développement.

Le nouveau code minier de la RDC de 2018 contient trente-deux innovations sur les opérations d'exploitation minière (demande d'exploitation minière, permis d'exploitation, étude d'impact environnemental sous l'approbation d'une Etude d'Impact environnemental et Social (EIES), capitaux des entreprises, etc.). Il contient aussi des obligations au plan social, sécuritaire et environnemental pour les exploitations minières. D'autres textes légaux tels que le code de l'urbanisme et de l'eau intègrent des exigences environnementales, loi relative à la conservation de la nature et des arrêtés ministériels en charge des mines, de l'administration territoriale et de la sécurité qui interdit l'orpaillage offensive sur l'environnement naturel et social, mais leurs applications posent des problèmes. Dans cette optique que nous réalisons les analyses ci-dessous tourné sur le projet d'exploitation minière semi - industrielle de la rivière Aruwimi.

\section{Maitrise du nouveau code minier de la RDC et droit des autochtones}

D'après le nouveau code minier de la RDC de 2018 et son décret $n^{\circ}$ 18/024 du 08 juin 2018, le capital social apporté par le requérant ne peut pas être inférieur à $40 \%$ des dites ressources. Et pourtant cette société chinoise Xiang Jiang Mining Sarl ne présente qu'un chiffre d'affaires de dix mille dollars américains. Les focus-groupes et les interviews semi- structurés organisés dans le territoire de Basoko auprès 742 personnes (locales et autochtones) révèlent la situation synthétisée dans le Tableau 1. Il ressort de ce tableau que 76,63 \% de la population enquêtée ne sont ni formées et ni sensibilisées sur le nouveau code minier de 2018. Les résultats obtenus démontrent que la population locale et peuple autochtone qui seront déplacés de leurs villages ignorent leurs droits. Ce qui viole le code minier dans son article 281, alinéas 7 et 7 bis.

Le tableau 2 renseigne que 79,5\% de la population locale et peuple autochtone enquêtés ne maitrisent ni la nouvelle loi, ni leur droit. Ils auront des obstacles juridiques pour rédiger le cahier des charges dans le cadre d'accord entre les deux partenaires (exploitant minière et la population locale) visant la concrétisation des actions du développement durable en vue d'améliorer leur bien-être social, économique et culturel (Article 285).

Tableau 1. Formation et sensibilisation des populations sur le nouveau code minier de 2018

\begin{tabular}{|c|c|c|c|c|c|c|}
\hline Catégories des interviews & \multicolumn{5}{|c|}{ Nombre et pourcentage de variable mesuré } \\
\hline Réponses & oui & $\%$ & Non & $\%$ & C.P & $\%$ \\
\hline Acteurs de la Société civile & 15 & 2,02 & 59 & 7,95 & 32 & 4,3 \\
\hline Chercheurs locaux et Etudiants & 26 & 3,5 & 51 & 6,87 & 29 & 3,9 \\
\hline Elèves des écoles secondaires & 2 & 0,27 & 97 & 13,1 & 7 & 0,9 \\
\hline Commerçants & 2 & 0,27 & 89 & 12 & 15 & 2 \\
\hline
\end{tabular}




\begin{tabular}{|c|c|c|c|c|c|c|}
\hline Pécheurs et Agriculteurs & 3 & 0,4 & 97 & 13,1 & 6 & 0,8 \\
\hline Autochtones et d'autres riverains & 6 & 0,80 & 88 & 11,9 & 12 & 1,6 \\
\hline Agents de l'administration territoriale & 10 & 1,34 & 89 & 12 & 7 & 0,9 \\
\hline Total & 64 & $\mathbf{8 , 6}$ & 570 & $\mathbf{7 6 , 8}$ & 108 & $\begin{array}{c}\mathbf{1 4}, \\
\mathbf{6}\end{array}$ \\
\hline
\end{tabular}

Source: Enquête sur terrain : janvier - juin 2019 et août - décembre 2020

Légende: C.P : Connaissance partielle

Tableau 2. Maitrise de la nouvelle loi et le droit de la population locale et peuple autochtone

\begin{tabular}{|c|c|c|c|c|c|c|}
\hline Catégories des interviews & \multicolumn{6}{|c|}{ Nombre et pourcentage de variable mesuré } \\
\hline Réponses & oui & $\%$ & non & $\%$ & C.P & $\%$ \\
\hline Acteurs de la Société civile & 18 & 2,4 & 57 & 7,68 & 31 & 4,2 \\
\hline Chercheurs locaux et Etudiants & 13 & 1,8 & 66 & 8,89 & 27 & 3,6 \\
\hline Les Elevés des écoles secondaires & 0 & 0 & 101 & 13,6 & 5 & 0,7 \\
\hline Commerçants & 0 & 0 & 96 & 12,9 & 10 & 1,3 \\
\hline Pécheurs \& Agriculteurs & 0 & 0 & 100 & 13,5 & 6 & 0,8 \\
\hline Autochtones et d'autres riverains & 9 & 1,2 & 88 & 11,9 & 9 & 1,2 \\
\hline Agents de l'administration territoriale & 16 & 2,2 & 85 & 11,5 & 5 & 0,7 \\
\hline Artisans de la Société civile & 56 & $\mathbf{7 , 5}$ & 593 & $\mathbf{7 9 , 5}$ & 93 & $\mathbf{1 3}$ \\
\hline
\end{tabular}

Source: Enquête sur terrain : janvier - juin 2019 et août - décembre 2020

Les mêmes interprétations viennent se confirmer par les analyses statistiques des régressions et $\mathrm{P}$ value $\left(\mathrm{R}^{2}=0,81\right.$ et $\mathrm{p}<0,05 ; \mathrm{R}^{2}=0,83 \mathrm{p}<$ 0,05 , figures 2 et 3 ). Il y a lieu de penser que, le comité permanent d'évaluation des activités minières ne se préoccupe pas de la formation de la population autochtone et locale qui va subir les conséquences après le passage de cette firme chinoise.

L’analyse de la Figure 4 montre que les Acteurs de la Société Civile semblent être plus impliqués dans la mobilisation du comité local sur les impacts environnementaux de l'exploitation minière semi - industrielle avec en moyenne $14 \pm 3,46$ d'intervalle.

Les peuples autochtones et les agents de l'administration locale (territoriale et de la chefferie) sont moins impliqués sur les conséquences d'impacts environnementaux de l'exploitation semi - industrielle et les résultats des analyses sont moins significatifs respectivement de $(8,83 \pm 3,48)$ et $(10 \pm 3)$ sur l'ensemble des données recueillies, alors que leur rôle est d'éclairer et d'éduquer la population sur la question d'impact environnemental et social des projets d'exploitation minière. En plus, les organisations de la société civile doivent accompagner les communautés locales en les avertissant sur leurs droits et leurs quotes-parts de la redevance minière, etc. 


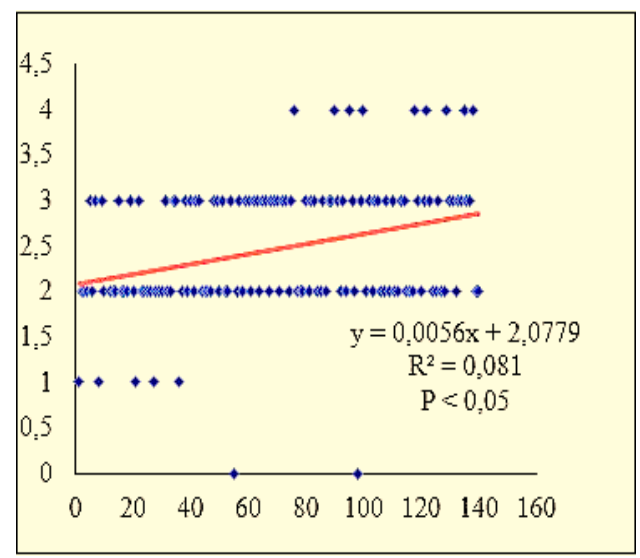

Figure 2. Réponse de P-value et régression sur la formation et sensibilisation sur le nouveau code minier de 2018

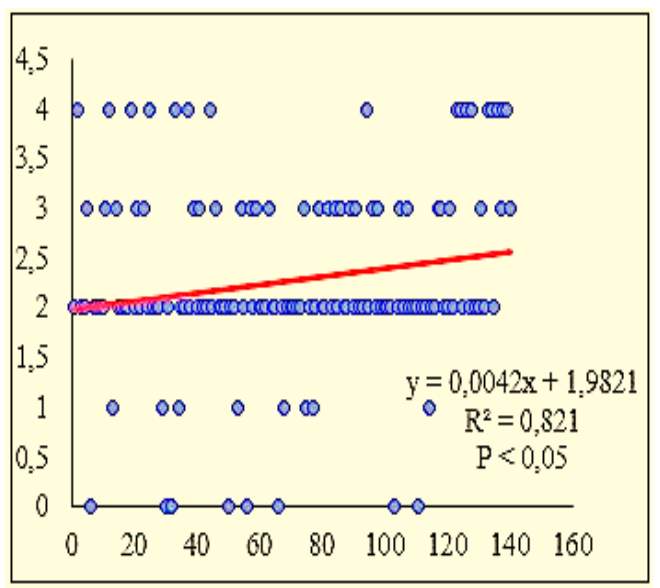

Figure 3. Réponse de P-value et régression sur la maitrise de la nouvelle loi et le droit de la population locale et autochtones

La dimension moyenne globale de l'analyse pour tous les acteurs montre d'une part que, le manque d'information et la non sensibilisation de ses acteurs sur le nouveau code minier de 2018 et la non maitrise de la nouvelle loi et le droit de la population locale et autochtones d'autre part n'est pas significative entre Catégories des interviews (Kruskal-Wallis : $\mathrm{X}^{2}=17.03$, df $=2$, $p$-value $=3.96 e-05)$. Les investigations ont montré que le nombre moyen des catégories des interviews n'est pas distinct d'un groupe à un autre. Ces alternances entraînent une différence significative entre les moyennes de différents groupes des catégories des interviews (Kruskal-Wallis : $\mathrm{X}^{2}=18.77$, $\mathrm{df}=2$, $p$-value $=3.117 e-07$. 


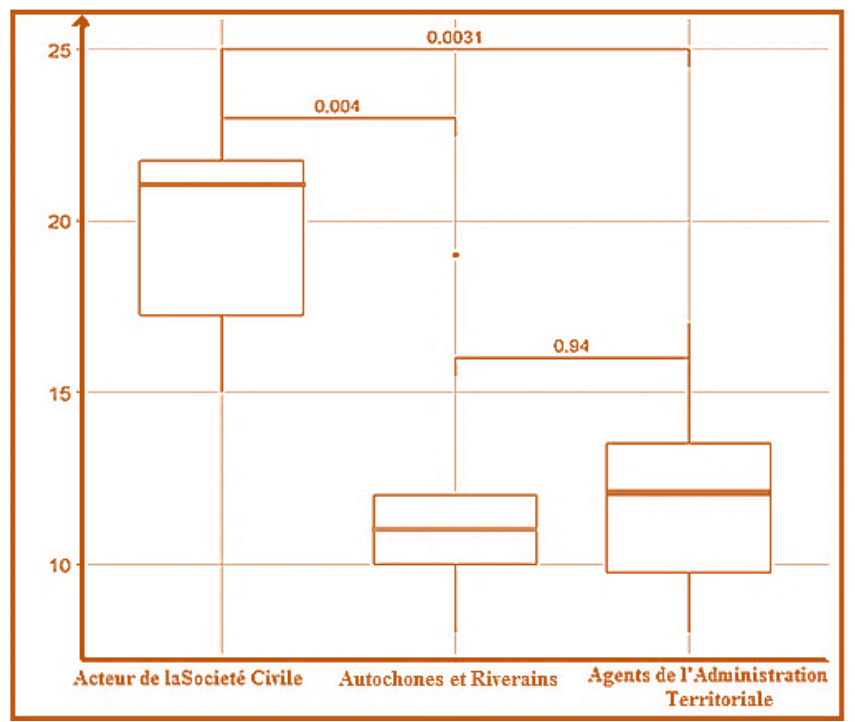

Figure 4. Distance de dimension d'importance des acteurs de comité permanent d'évaluation des activités minières

Ces résultats montrent que les textes légaux en vigueur dans notre pays présentent l'exigence d'évaluation environnementale et sociale pour ce type d'activité (exploitation minière) mais force est de reconnaitre que cet outil qui devrait permettre de prévenir et gérer les risques et les catastrophes, n'est pas convenablement utilisé ou reste ignoré par certains acteurs.

\section{Exploitation Minière : une activité sous pression environnementaliste Panorama de l'exploitation minière et les pratiques locales de survie}

L'agriculture et la pêche sont les activités les plus pratiquées par la population de Basoko, à cause des habitudes culturelles des tribus, mais dans principe de la productivité des ressources (sol, forêt et eau). Voici les propos d'un notable: "l'eau est pour nous l'élément essentiel pour notre vie des Basoko dans notre diversité culturelle. Notre vie ne se conçoit pas et ne se vit pas sans rivière, ruisseau ou fleuve. L'exploitation minière sur notre rivière perturbera notre vie quotidienne, malgré les multiples promesses de développement. » Un autre déclare : "cette exploitation ne nous permettra plus de produire riz, arachides, huiles de palme, maïs, ignames, bananes, maniocs etc., sur les terres côtières de la rivière Aruwimi. Nous vivons de cette forêt marécageuse que traverse notre rivière qui nous produit les gibiers, les escargots, les chenilles, les termites, etc. Et la pêche est notre activité quotidienne privilégiée. Nos enfants ne mangeront plus des poissons frais, fumés, salés... ainsi que les produits de l'élevage domestique (poules, porcs, chèvres, moutons...» 


\section{Regards sur les impacts environnementaux : Discours de la population locale}

Le projet minier sur la rivière Aruwimi dans la zone de Liambe (Baonde, Liambe, Yakoyo, Yangonde-Lioto, Ilongo, Likombe, Bobaula, Mongandjo...) visant l'exploitation semi-industrielle des gisements aurifères et adamantins est à haut risque pour plus de $80 \%$ de la population souligne un leader local. D’autres témoignages enregistrés élucident que «Depuis des générations, les riverains de cette majestueuse rivière vivant principalement des fruits de ses eaux et en usent comme boisson. Et cette rivière servant de colonne vertébrale pour ces communautés et la principale voie de communication pour leur mobilité. ». "Si jamais une exploitation minière avait lieu dans Aruwimi, les effets sur la qualité de l'eau et la disponibilité des ressources en eau dans la zone du projet en constitueraient, peut-être, l'impact le plus important ».

Les questions clés seraient de savoir si la qualité des eaux, les apports en eau de surface resteraient propices à la consommation humaine et supporteraient la vie aquatique et la faune terrestre native dans la zone du projet. Selon la ligue des chefs coutumiers : «Liambe étant dans une zone à forte pluviométrie, des facteurs de pollution des eaux peuvent se produire par le secteur artisanal minier à petite échelle locale et informelle, figure 5». Les conséquences seront énormes pour la région d'exploitation et au-delà de l'embouchure de la rivière Aruwimi. Dans les eaux de surface, des concentrations élevées de particules dans la colonne d'eau pourront produire des effets toxiques à la fois chroniques et aigus chez les poissons qui sont une des Ressources à la base des Activités Régénératrices des Revenues (AGR) et la principale source protéinique des riverains.

L'exemple de Banalia, en amont de la rivière Aruwimi nous renseigne selon le rapport de SAESSCAM- Antenne de Kisangani (2020) que : " Les grandes quantités d'eau qui seront déversées dans la nature, durant toute la durée de l'exploration, contenant des débris de roches du sol 'argilosablonneux', auraient des impacts fâcheux sur la vie végétale et même animale dans la région déversoire. Donc, nous sommes contre cette exploitation, nous les détenteurs de la terre et des eaux ancestraux ».

D'après le même rapport de SAESSCAM - Antenne de Kisangani (2020), toutes exploitations minières semi-industrielle vécues dans la province de la Tshopo provoquent des dépôts des sédiments dans les eaux de surface, ou dans les plaines d'inondation d'une vallée de cours d'eau et aggravera davantage les processus d'érosion. La sédimentation causera l'accumulation de couches épaisses de minéraux fins et d'alluvions dans les endroits d'inondation ainsi que la putréfaction des habitats aquatiques et la perte de capacité de stockage des plans d'eau. Le cas de nos voisins, les Abolo de Banalia en est une illustration. 
Dans le cas de la zone de Liambe et ses environs, vue son sol argilosablonneux et érosif, les principaux facteurs qui influenceront l'érosion comprennent le cubage et la promptitude du ruissellement provenant des précipitations, le taux de pénétration dans le sol, la quantité de la couverture végétale qui sera détruite, la distance de la pente ou l'intervalle à partir du point d'origine de l'écoulement en nappe ainsi que les compositions de contrôle d'érosions opérationnelles.

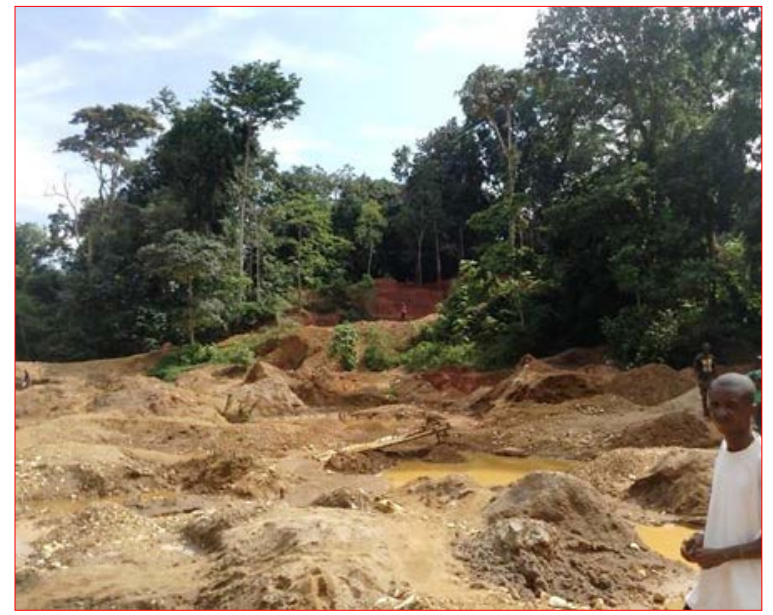

Figure 5. Terrain frivole et accidenté résultat du secteur artisanal minier à petite échelle locale sur la berge de la rivière Aruwimi aux environs de Liambe (Photo Dewe, 2020)

\section{Déforestation et dégradation des sols et pollution atmosphérique Déforestation et dégradation des sols}

Avant l'exploitation minière, il faut aussi voir l'aspect agricole et comprendre l'évolution du mécanisme de déforestation durant les années à venir. Un chercheur de la lignée royale nous démontrera que les impacts de déforestation et de dégradation des sols liés à l'exploitation minière semiindustrielle. « D’après lui, ces activités minières sont des véritables catalyses de dégradation profonde des conditions bio- et pédoclimatiques qui pourraient entraîner un autre régime thermique ainsi qu'une nouvelle dynamique de l'eau dans le sol et affecteront les premiers mètres du profil pédologique. Ce fut le cas à Banalia où l'exploitation ne se faisait que sur 10 carrés miniers ».

Pour Basoko, 50 carrés miniers seront mis en exploitation ($\mathrm{N}^{\circ} \mathrm{CAM} / \mathrm{CR} / 8262$ du 29 novembre 2019 pour le Permis de recherche N¹4764, Exclusivité de 30 carrés, situés dans le territoire de BASOKO en Province Orientale et $\mathrm{N}^{\circ} \mathrm{CAM} / \mathrm{CR} / 8261$ du 29 novembre 2019 pour le Permis de recherche $\mathrm{N}^{\circ} 14763$, Exclusivité de 20 carrés, situés dans le territoire de BASOKO en Province de la Tshopo). Les résultats de l'exploitation minière locale (exploitants locaux) dans l'Hinterland de la rivière Aruwimi mettront 
en cause la stabilité de la structure modifiée par la dégradation des propriétés physiques notamment : la porosité, la densité, la perméabilité, l’infiltration, l'érosion qui appauvrissent le sol en particules fines, le réel support à la fertilité du sol ; l'appauvrissement du stock organique (ce qui rompt l'équilibre minéralisation - humification) ; l'acidification et enfin les déséquilibres minéraux (Figure 6).

\section{Les émissions atmosphériques des subsistances nuisibles}

Les émissions atmosphériques se produisent à chaque étape du cycle de la mine, mais surtout pendant l'exploitation, le développement, la construction et les activités opérationnelles, en réduisant la pollution en fin de cycle, déclare un scientifique : " on risque de la transférer d'un milieu à un autre, où elle pourrait créer des problèmes environnementaux tout aussi graves, ou même finir par être une source indirecte de pollution du même milieu. Sans être aussi onéreuse qu'une mesure correctrice, la réduction de la pollution en fin de cycle peut augmenter sensiblement le coût des procédés de production sans ajouter de valeur " suivant le rapport de SAESSCAM (Service d'assistance et d'encadrement du secteur minier de type artisanal) de Kisangani.

L'exemple de Banalia et de Bafwasende nous renseigne par SAESSCAM- Kisangani que: "les opérations minières requièrent de grandes quantités de substances et des déchets contenant des particules qui sont souvent dispersées par le vent et sont souvent sources de la contamination atmosphérique dans les opérations minières».

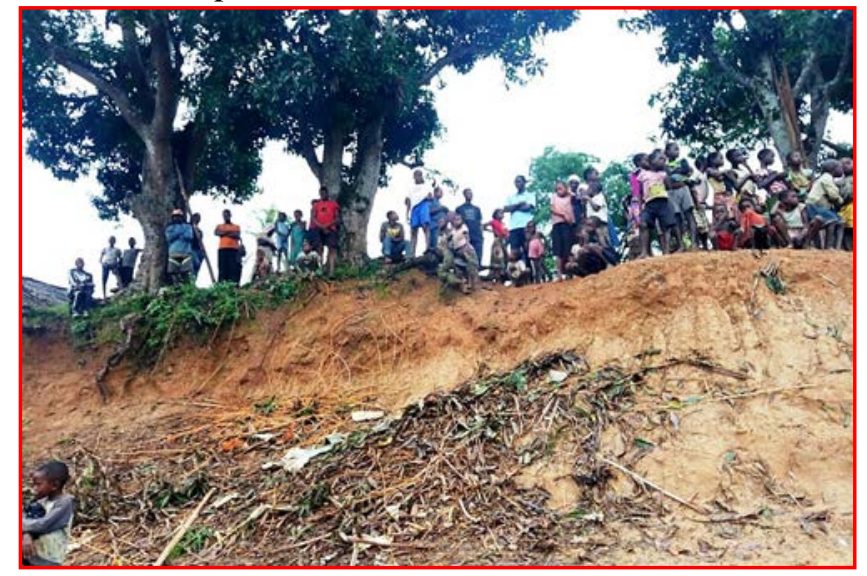

Figure 6. Destruction de verge de la rivière Aruwimi aux environs de la Localité de Likombe, Territoire de Basoko (Photo M. Silencieuses, 2009)

Un agronome du milieu, en se basant du même rapport de SAESSCAM- Kisangani, dit que : "ces émissions des particules dans l'atmosphère comprennent : le stockage et le transport de matériaux, le 
traitement des mines, la poussière fugitive, l'abattage, les activités de construction et les galeries associées aux activités minières, les coussins de lixiviation, les tas de résidus de minerais, les bassins de transvasements et les amas de déchets des roches. Les sources et les caractéristiques des émissions des poussières fugitives dans les opérations minières varient dans chaque cas, il en est de même pour leurs impacts. Les particules des matières sont transportées par le vent, à la suite de fouilles d'abattages par explosion, de transport des matériaux, de la désagrégation par le vent, des particules fugitives provenant des installations des résidus, des endroits de culbutage...qui provoquent des effets graves sur la santé humaine (maladie d'origine cardiaque et pneumonie) et sur l'environnement ".

A tous les niveaux, les effets sur l'environnement seront perceptibles. Ces exploitations présenteront des risques sur l'environnement. En autre terme, l'exploitation minière est une des activités ayant le plus fort impact environnemental et à ce titre elle est fortement contestée par les ONG environnementalistes et les communautés locales qui en subissent directement les effets de la pollution, de la dégradation de l'environnement, les pollutions et des nuisances.

\section{Exploitation Miniere Et Defis Environnementaux : Diversite Des Situations}

La gestion des terres et des ressources naturelles compte parmi les défis critiques auxquels les pays en développement sont confrontés aujourd'hui. L'exploitation de ressources naturelles de forte valeur, qu'il s'agisse d'hydrocarbures, de minéraux ou de bois, a souvent été associée à la genèse, à l'escalade ou à la persistance de conflits violents autour des firmes étrangères et la population locale. On observe aussi une multiplication des situations de concurrence et de conflit face à une diminution de ressources renouvelables, notamment en eau, minerais et en terres (Hanson, et al., 2009).

Cet état de choses a été encore aggravé par la dégradation de l'environnement, la croissance démographique et le changement climatique. La gestion défectueuse de terres et de ressources naturelles a contribué à la création de nouveaux conflits, tout en compromettant la résolution pacifique de ceux qui existaient déjà (Hanson, et al., 2009). Ainsi, les divers champs d'application de l'évaluation environnementale dans la prévention et la gestion des catastrophes, tant au niveau de la réalisation des projets miniers qu'à celui de la planification stratégique permettant de faire le bilan des pratiques à cet égard, semblent méconnus ou mal appliqués. 


\section{Demande mondiale des minerais}

L'essor considérable de la demande mondiale de certains minerais depuis la fin du $X^{\text {ème }}$ a conduit à la multiplication des grands projets miniers, concernant notamment des métaux très convoités et de plus en plus rares comme l'or. Dans le même temps, les firmes qui veulent créer de nouvelles exploitations minières de grandes dimensions et à l'impact environnemental considérable, risquent de plus en plus de se heurter à une opposition résolue des populations locales soutenues par des ONG environnementalistes. L'influence de ces dernières sur les opinions publiques par le biais de campagnes médiatiques, peut faire pression sur les Etats et amener ceux-ci à remettre en cause les projets miniers (Wakenge, 2019).

Certaines firmes, conscientes de l'enjeu capital que constitue leur image environnementale, ont réussi, mieux que d'autres, à développer une stratégie, une communication et des actions permettant de rendre plus acceptables leurs projets miniers, même quand ceux-ci ont un impact considérable sur l'environnement (Bebbington et al., 2008). Si les conflits entre compagnies minières et groupes environnementalistes ont été de plus en plus nombreux au cours des dernières années, notamment dans les pays en voie de développement, ils ne sont pas non plus systématiques et n’ont pas reçu le même soutien des communautés d'habitants (Coulibaly, 2013). L'analyse de différents projets d'exploitations aurifères et diamantifères en cours, ou au contraire infructueux, permet d'étudier la stratégie suivie par les firmes pour se rendre environnementalement acceptables. Elle permet aussi de discuter de l'impact de ces projets à plus ou moins long terme dans la perspective du développement durable (Glasson \& Therivel, 2013).

\section{Minerais de conflit: malédiction des ressources naturelles et/ou le développement?}

Si la région africaine des Grands Lacs possède d'énormes richesses naturelles, on en parle plus souvent en termes de "malédiction des ressources naturelles » et "minerais de conflit» plutôt qu'en termes de "développement, emploi et soulagement de la pauvreté".

La situation difficile dans l'Est de la RDC suscite une attention croissante de la part de la communauté internationale. L'exploitation des minerais du Congo se déroule actuellement dans un climat d'insécurité, de financement de groupes rebelles, de violation des droits humains, de taxation illégale et de contrebande, privant ainsi l'Etat des moyens financiers nécessaires (Matthysen et al., 2011).

L'exploitation minière consiste à extraire des pierres de la croûte terrestre contenant des minéraux ou des métaux utiles en proportion suffisamment intéressante pour justifier leur exploitation (Matheus, 2018). Durant cette exploitation, la gestion de l'eau, de l'énergie et des déchets 
constitue une part importante qui détruit l'environnement, les déchets générés sont potentiellement dangereux et ses conséquences environnementales se maintiennent sur la productivité des sites et ont des effets d'héritage non voulus jusqu'aux générations futures (Matheus, 2018 ; Wakenge, 2019). Nonobstant le durcissement de la réglementation environnementale et le nouveau code minier, la pression accrue de l'opinion publique proteste contre l'exploitation (Wakenge, 2019), des mines du lit de la rivière Aruwimi dans la zone de Liambe, malgré l'obtention des permis d'exploitation par une société chinoise Xiang Jiang Mining Sarl.

En amont de la rivière, cette société chinoise avait extrait de l'or et de diamant. Malheureusement à l'ouverture, pendant l'extraction et à la fermeture de la mine, cette société n'avait pas développé des stratégies pour réduire les risques, et mettre en place de meilleures pratiques pour diminuer les coûts d'assainissement environnemental. Au contraire, les pollutions, la destruction environnementale et la non coopération avec les communautés locales sont les souvenirs que la société aurait laissé dans le territoire de Banalia (Ekele \& Mangambu, 2020).

Face à l'obstination de cette société d'exploiter la rivière Aruwimi avec ses permis de recherches, malgré l'opposition de la communauté locale, cette société cherche toujours des moyens et des soutiens politique avec la complicité de certaines notabilités pour une exploitation forcée sans tenir compte des patrimoines centrales même si le sol et le sous-sol appartiennent à l'Etat. Si les populations de Basoko, particulièrement celles de la zone d'exploitation sont expropriées de leur terre au profit de cette société, elles seront de toute évidence, privées de leurs principaux moyens de subsistance.

\section{L'essor de l'exploitation minière: un privilège majeur pour le développement du pays?}

L'essor de l'industrie minière est une prérogative pour la RDC, la connaissance des stratégies et des palliatifs pour juguler les dégâts éventuels est capitale. Cela permettra à l'ayant droit, c'est - à-dire à la communauté locale de prendre une décision éclairée et responsable. En RDC, les fondations légales concernant la protection et la gestion de l'environnement dans le cadre des activités minières sont fondées sur différents textes réglementaires, dont le code minier, établi en 2002 et révisé en 2018 qui malheureusement ne sont pas respectés. Ce code minier révisé devrait conditionner l'octroi d'un permis d'exploitation à la préparation et la réalisation préalable d'une prospection des études d'impacts environnementaux (EIE) etc. Les réponses reçues durant les investigations montrent que la population locale n'est pas informée de ses droits mais est bien informée de ce qui était survenu à Banalia et Bafwasende, territoire voisin de Basoko. Cette population a peur des conséquences qui se 
lisent en filigrane sur les impacts enregistrés à Banalia, à la suite d'une exploitation similaire.

Au lieu donc de tenir compte de l'intérêt du peuple congolais et de l'avenir des générations futures, les gouvernements national et provincial ont préféré vendre quasiment la moitié de cette rivière dans le territoire de Basoko, la découper en carrés miniers, soit pour un contrat de recherche, soit pour un contrat d'exploitation. Ne comptant que sur le présent, le gouvernement congolais démontre le manque de la politique de planification à court, à moyen et à long terme pour assurer aux générations futures d'un cadre idéal pour leur épanouissement et leur développement. Avec cet esprit affairiste et mercantiliste dans le patronat de nos autorités (certaines autorités politiques de la province, des Entité Territoriale Décentralisée, cadres du cadastre minier ont poussé à l'extrême sur l'enrichissement facile à travers le secteur minier) qui caractérise cette diplomatie, les germes de futurs conflits terriens entre les grands propriétaires miniers et le petit peuple congolais sont bien perceptibles. L'eau et la terre sont des causes des grands conflits partout au monde ; croire qu'on peut les résoudre soit par pression, soit par l'argent, relève de l'illusion et de manque de sens de prévention pour l'avenir.

\section{Impacts sur les ressources en eau}

Reliée à la question de la fertilité des sols et, par conséquent, à la survie et au bon fonctionnement de toutes les sociétés humaines depuis leurs origines, l'eau douce occupe une place centrale dans les préoccupations quotidiennes des hommes et fait l'objet d'une fervente adoration dans de nombreuses cultures. La forme que revêtent les eaux porteuses de bienfaits varie en revanche en fonction des sociétés et de leur environnement. L'importance vitale de l'eau douce la fait figurer dans certains mythes de la création. Dans les croyances mésopotamiennes l'eau douce, est à l'origine de tout ; et est généralement liée aux mythes concernant l'origine de l'espèce humaine. La mythologie égyptienne ancienne, dont la prospérité est liée aux crues du Nil qui fertilisent les terres de la vallée qui s'étend en plein désert, plusieurs divinités sont directement associées au fleuve (Glasson \& Therivel, 2013).

\section{Le drainage d'acide minier et la lixiviation des contaminants}

La lixiviation (du latin lixivium=lessive) est la méthode d'extirpation de minerai par lavage et percolation, pour le cas d'espèce, à l'acide sulfurique (Lu, 2012). La capacité de drainage de l'acide minier est une question-clé. La réponse déterminera si ce projet minier dans la région de Liambe est acceptable pour l'environnement. Lorsque les matériaux minés sont creusés, et exposés à l'eau et à l'oxygène, des acides peuvent se former si les minéraux sulfure ferreux sont nombreux et s'il y a une capacité approximative de matériaux neutralisants pour nettoyer André et al. (2006) et Rapport de 
SAESSCAM, Antenne de Kisangani (2020). Pour compenser l'acide, à son tour, lessivera ou dissoudra les métaux et autres contaminants dans les matériaux minés et formera une solution acide, à forte teneur en sulfates et riche en métal (Vaessen \& Brentführer, 2015).

\section{L'érosion des sols et les déchets miniers dans les eaux de surface}

Le sol de Liambe et de toute la zone environnante où le projet minier voudrait s'implanter est argilo-sablonneux et subit des érosions régulières. Cette condition de ravinement du sol, la formation de boues dans l'eau de surface et la dégradation de la qualité de celle-ci est un grave problème de la région. La pédologie de ce bassin de sédimentation " argilo-sablonneux » qui fait partie de la cuvette centrale est émergée «d'une mer intérieure » très riche en tourbières. Ces capteurs de gaz à effet de serre (les tourbières) dont la libération du CO2 (emprisonné dans les tourbières) aura des effets nuisibles pour toute la planète, à la suite du réchauffement climatique qui en résulte.

Une étude financée par la Commission Européenne dans le Katanga et le Kasaï, a révélé que la grande superficie de terre est endommagée par l'exploitation minière et par des grandes quantités de substances exposées sur les sites. Le ravinement du sol est une préoccupation majeure sur les sites miniers (Glasson \& Therivel, 2013). La lutte contre l'érosion doit être de mise, depuis le début des opérations jusqu'à l'achèvement des travaux de réhabilitation environnementale (Otto, 2009 ; Vaessen \& Brentführer, 2015).

Le sol de Liambe est de nature érosive, toute exploitation de genre minier pourrait provoquer des chargements importants des atterrissements vers des plans d'eau proches, pendant des tourmentes sévères et de grandes périodes de la crue d'eau de la rivière Aruwimi (Figure 7). La population de Liambe jusqu'à Yakoyo serait obligée d'émigrer, mieux encore de se délocaliser. Vue son sol argilo-sablonneux et érosif de la zone de Liambe, Likombe, Ilongo-Mbutu, Ilongo-Koki, Yangonde-Lioto, Yakoyo, Bomane, Baonde,...les principaux facteurs qui influenceront l'érosion comprennent le volume et la vitesse du ruissellement provenant des précipitations, le taux d'infiltration des précipitations dans le sol, la quantité de la couverture végétale qui sera détruite, la longueur de la pente ou la distance à partir du point d'origine du ruissellement en nappe au point où la sédimentation commence, ainsi que les structures de contrôle d'érosion opérationnelles (Vaessen \& Brentführer, 2015).

Les incidences pourraient inclure une baisse $\mathrm{du} \mathrm{pH}$ ou des accumulations des métaux dans les eaux de surface et/ou la contamination persistante des sources d'eaux souterraines. Les sédiments contaminés peuvent également réduire le $\mathrm{pH}$ des sols, de telle sorte que la végétation et les habitats qui s'y rattachent soient effrités au point d'entamer la productivité 
agricole et créer des perturbations climatiques au niveau local (Busse \& Gröning, 2013).

Au-delà du potentiel pour des impacts polluants sur la vie humaine et aquatique, il existe des impacts physiques potentiels associés à l'augmentation du volume et de la vitesse de ruissellement découlant des nouvelles activités de perturbation des terres. Des vitesses et volumes accrus peuvent conduire à des inondations en aval, l'érosion des canaux de cours d'eau et des dommages structuraux au niveau des pieds des ponts et des entrées des ponceaux (Stratos, 2011).
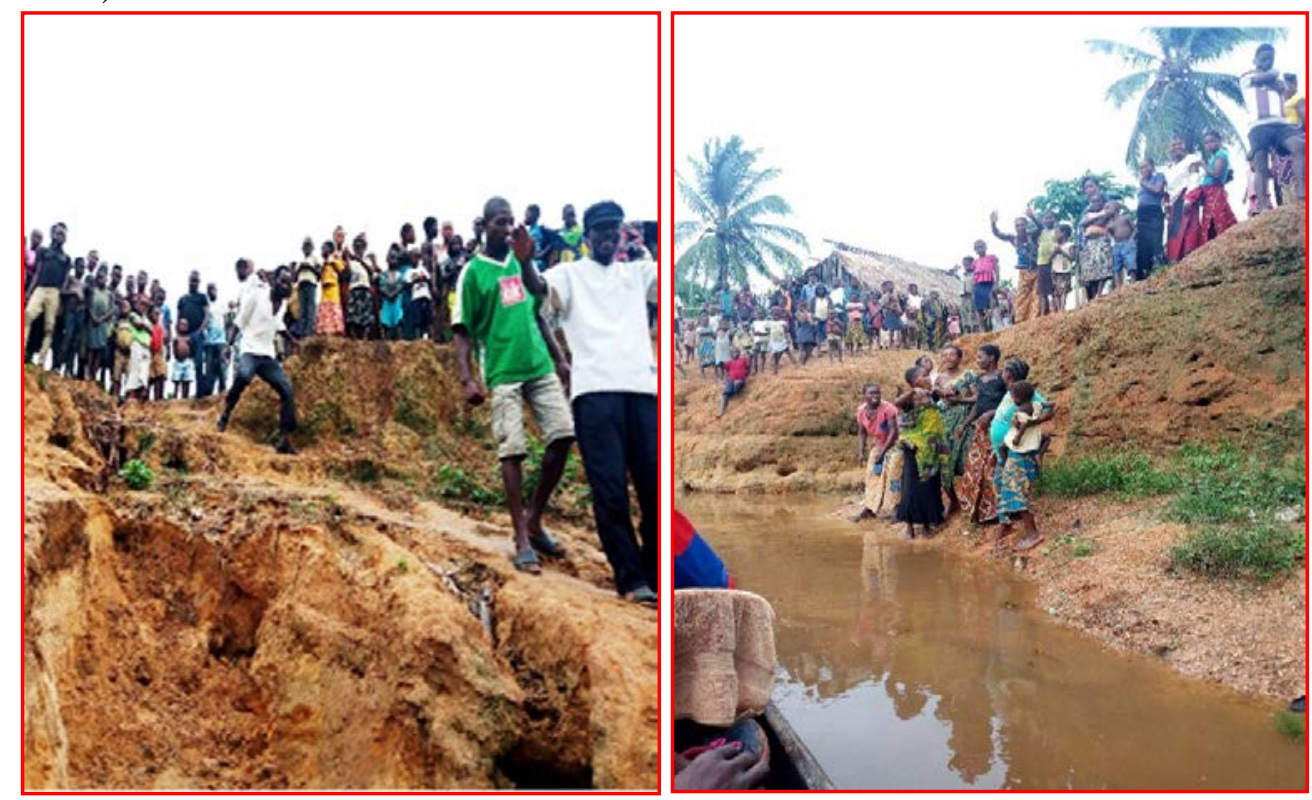

Figure 7. A Gauche, terrain superficiel du village Yakoyo, et à Droite, terrain superficiel du village Baonde, riverain de la rivière Aruwimi (Photo Dewe)

Une observation scientifique laisse penser que dans la zone de Liambe et ses environs (Likombe, Ilongo-Mbutu, Ilongo-Koki, Yangonde-Lioto, Yakoyo, Bomane, Baonde..), les émissions atmosphériques ont déposé des particules acides où la végétation naturelle a été détruite. Le ruissellement a le potentiel d'augmenter le taux d'érosion et de conduire à la suppression du sol de la zone concernée. Cette hypothèse repose sur son paysage caractérisé par des pentes rocheuses et en escaliers. Une fois que le sol sera érodé par les effets d'exploitation décrits ci-haut, la végétation de la pente se reconstituera difficilement, que ce soit naturellement ou avec assistance humaine.

Impacts des bassins de décantation des résidus, de déchets de roche, de la lixiviation en tas et des installations de stockage de lixiviats

Les impacts des bassins de décantation des résidus de mine, des déchets de roche, de la lixiviation en tas et des installations de stockage de 
lixiviats sur la qualité de l'eau pourront aussi avoir des conséquences graves dans la zone de Liambe. Ces effets comprennent la contamination des eaux souterraines en dessous de ces installations et des eaux de surface. Les substances toxiques peuvent alors lessiver ces installations, filtrer à travers le sol et contaminer les eaux souterraines, surtout si la base de ces installations n'est pas équipée d'un revêtement imperméable (Vaessen \& Brentführer, 2015).

D’après ACI - RDC (2009), Au Katanga les terrils (sous-produit du traitement de minerai métallique) représentent de grosses quantités de déchets qui peuvent contenir des volumes des substances toxiques dangereux, telles que l'arsenic, le plomb, le cadmium, le chrome, le nickel et le cyanure (si la lixiviation au cyanure est utilisée). Le constat est que la plupart des firmes étrangères minières de la $\mathrm{RD}$ Congo ne dégagent pas des terrils en les mélangeant avec de l'eau (pour former une pulpe) et maintiennent cette pulpe dans un grand bassin de décantage de résidus de mine ACI -RDC (2009). Vu que le minerai est généralement extrait comme une pulpe, les résidus qui en découlent renferment de énormes quantités d'eau et souvent forment des étangs tout au sommet des bassins de déchets et peuvent constituer donc une menace pour la faune et une cause des maladies d'origine diverses, souvent hydrique (ACI -RDC, 2009). Plus grave et horrible, lorsque les bassins de décantation de déchets de mine concèdent, ils déversent de grandes quantités d'eaux dangereuses qui peuvent tuer la vie aquatique et empoisonner l'approvisionnement en eau potable sur de nombreux kilomètres en aval du bassin de l'épuration (Otto, 2009 ; Vaessen \& Brentführer, 2015).

\section{Impacts d'exploitation des mines à ciel ouvert}

Lorsqu'une mine à ciel ouvert, comme prévu dans le contrat du projet minier d'exploitation minière par la Societe Xiang Jiang Mining SARL dans le territoire de Basoko, l'intercepte, la nappe phréatique et les eaux souterraines envahissent le puits. Pour poursuivre les travaux d'exploration, les compagnies minières doivent pomper cette eau vers un autre endroit. Le pompage et le déversement des eaux provoquent des conséquences environnementales qui sont décrites dans une étude commanditée par la Commission européenne menée au Katanga et au Kasaï (Agir en Chrétiens Informés, ONG, 2009).

L'eau peut être pompée à partir des puits entourant la mine pour créer un cône de dépression dans le niveau de la nappe pour réduire l'infiltration Coleman J. (2018). Lorsque la mine est opérationnelle, l'eau doit être constamment retirée pour faciliter la récupération du minerai. Lorsque les opérations minières prennent fin, le pompage et la gestion de l'eau de mine s'arrêtent aussi, ce qui entraîne une possible accumulation dans les fractures des roches, des puits, des tunnels, de puits à ciel ouvert et des rejets 
incontrôlés dans l'environnement et les impacts pourraient durer plusieurs décennies (Vaessen \& Brentführer, 2015).

\section{Impacts de projets miniers sur la qualité de l'air Rejets fortuits de mercure}

Dans le projet d'exploitation de la zone de Liambe, il est prévu l'exploitation de l'or et de diamant. Le mercure est généralement présent dans les minerais d'or et dans les déchets associés et les concentrations varient considérablement, même au sein d'un gisement spécifique. D’après les études menées par les chercheurs de l'Université du Burundi (2019), la teneur en mercure dans un minerai d'or est de $10 \mathrm{mg} / \mathrm{kg}$ et un million de tonnes de minerai sont traitées dans un site minier particulier (des concentrations pas inhabituelles). Ces études signalent que 10 tonnes de mercure sont potentiellement rejetées dans l'environnement chez les Badro Manning au Sud-Kivu ; l'or s'avère comme une source majeure de mercure qui doit être contrôlée (PNUD, 2016).

\section{Bruits et vibrations}

L'impureté par le bruit (Figure 8) associé à l'exploitation minière implique les bruits en provenance des moteurs des engins, le chargement et le transport de roches dans des torrents en acier, les traineaux, la production électrique etc. Les impacts complémentaires des pelles mécaniques, du recadrage, de la prospection, de l'abattage par explosion, du transport, du concassage, du broyage et du stockage en grands volumes peuvent affecter de manière significative la faune et la population riveraine. La vibration affecte l'équilibre des infrastructures, la stabilité des bâtiments et des maisons et/ou les domiciles de la population riveraine des travaux des grandes mines à ciel ouvert. Une étude parrainée par l'Union européenne en 2010, les résultats montrent que dans les mines sous exploitants Chinois, les outils utilisés créent des chocs et les vibrations, à la suite d'abattages en relation avec l'exploitation minière peuvent entraîner du bruit, de la poussière et conduire à la destruction des structures dans les zones environnantes non-habitées.

\section{Impacts des projets miniers sur la biodiversité et perte d'habitat}

Le degré d'atténuation est une approche fréquentative s'appuyant sur les bonnes pratiques internationales, qui vise à limiter et à gérer les impacts inappropriés de l'exploitation minière, afin d'aligner les besoins environnementaux et sociaux avec les priorités de l'essor de la durabilité (Lanmafankpotin, et al., 2013). L'exploitation minière a un choc sur le milieu et les biotopes associés par le biais de la disparition de la végétation ainsi que le sol superficiel, le déplacement de la faune, le dégagement de polluants et la génération de bruit (Kemp, 2009 ; Lanmafankpotin, et al., 2013). La perte 
d'habitat naturel est l'un des impacts majeurs des projets miniers sur la biodiversité. Les mines à ciel ouvert provoquent du débroussaillage et donc la perte directe d'habitats naturels.

Les impacts indirects découlant de l'immigration de personnes à la recherche d'une meilleure situation économique peuvent également aggraver la perte d'habitats (et leur dégradation). Les espèces de la faune vivent dans des communautés qui dépendent les unes des autres. La survie de ces espèces peut dépendre des conditions du sol, du climat local, de l'altitude et d'autres caractéristiques de l'habitat local. L'exploitation minière de Liambe et ses environs pourrait provoquer des dommages directs et indirects sur la faune locale et voisine. Les impacts proviennent principalement de la perturbation, du déplacement et de la redistribution de la surface du sol. Certains impacts sont de court terme et sont limités au site de la mine; d'autres peuvent avoir des répercussions profondes et des effets de long terme (Ekele \& Mangambu, 2020).

L'effet le plus direct sur la faune est la destruction ou le déplacement des espèces dans les zones de cavité et d'entassement des dépôts des résidus miniers. Les espèces mobiles de la faune, comme le gibier, les oiseaux et les prédateurs, abandonnent ces zones. Les animaux plus sédentaires, comme les invertébrés, de nombreux reptiles, les rongeurs fouisseurs et les petits mammifères, peuvent être plus sévèrement affectés (Mangambu, 2021 sous presse). On pense que pour la rivière Aruwimi, les poissons, les invertébrés aquatiques et les amphibiens seront sévèrement touchés et tous les minerais seront détectés et ramassés par détecteur aurifère installé dans dragues robots (Figure 8). L'approvisionnement en nourriture des prédateurs est réduit par la disparition des espèces terrestres et aquatiques. De nombreuses espèces de la faune sont fortement dépendantes de la flore grandissant dans les drainages naturels (Busse \& Gröning, 2013). La végétation fournit les aliments indispensables, les sites de nidification et des abris pour échapper aux prédateurs (PNUD, 2012).

Toute activité qui détruit la végétation près des étangs, des réservoirs, des marais et des marécages réduit la qualité et la quantité de l'habitat essentiel pour les oiseaux aquatiques, les oiseaux de rivage et de nombreuses espèces terrestres (Kemp, 2009). Les exigences de l'habitat de nombreuses espèces animales ne leur permettent pas de s'adapter aux changements créés par la perturbation du terrain. Ces modifications réduisent l'espace vital. Le degré auquel les animaux tolèrent la concurrence humaine pour l'espace varie (Busse \& Gröning, 2013). Certaines espèces tolèrent très peu de changement. Dans le cas où un milieu spécifique ou une zone de reproduction initiale devient limité, une espèce pourrait disparaître. Les mines à ciel ouvert comme prévu dans le cas de Liambe, peuvent dégrader les habitats aquatiques avec des impacts ressentis à de nombreux kilomètres du site minier (Ashanti 
Goldfields Kilo, 2015) ; la contamination des sédiments de la rivière Aruwimi et de cours d'eau est courante avec l'exploitation à ciel ouvert.
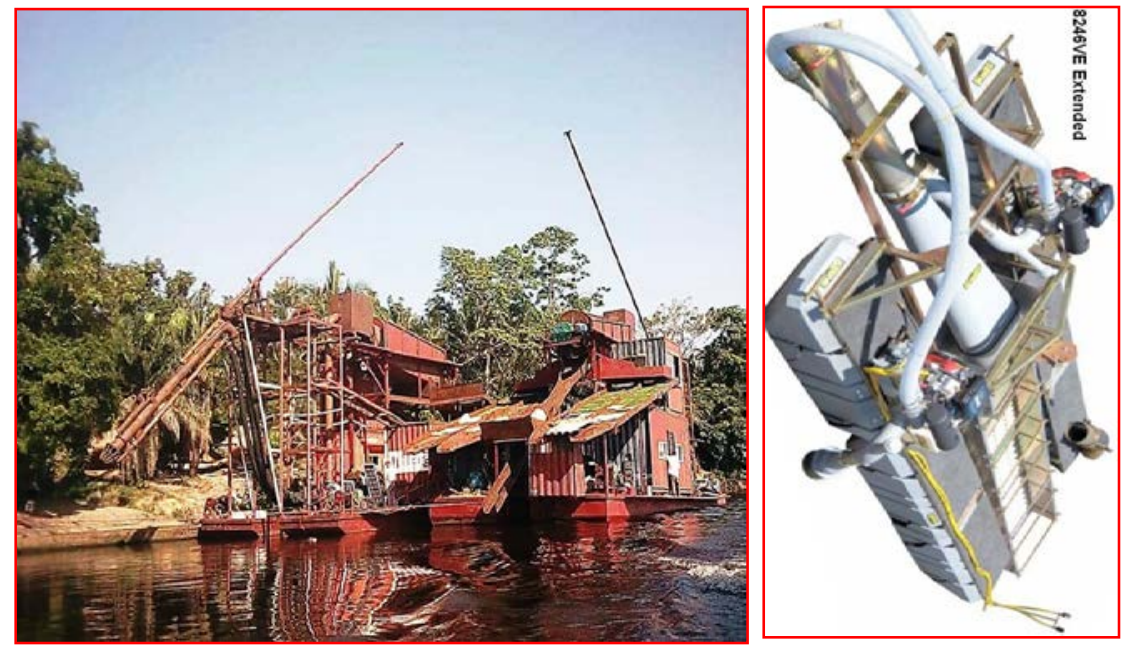

Figure 8. A droite, les dragues robots « Une drague est un dispositif installé sur un bateau ou un radeau, qui opère sur les rivières pour extraire des particules » de la société « Xiang Jiang Mining SARL » dans le port de Yangoma, territoire de Basoko et A gauche

Détecteur aurifère installé dans dragues robots de la société de la Xiang Jiang Mining SARL (Photo M. Silencieuse, 2020)

\section{Dégradation et appauvrissement des habitats naturels}

Les activités minières peuvent réduire la qualité des habitats de plusieurs manières. Par exemple, la qualité des habitats aquatiques peut être détériorée en raison du drainage minier acide, de la contamination du sol, de l'atterrissement et du rejet de résidus non-traités. Cela peut à son tour avoir des effets toxiques sur les plantes et les animaux, y compris les poissons et les oiseaux. Ces impacts peuvent également s'étendre aux écosystèmes terrestres de la zone d'exploitation. La poussière provenant des activités minières peut également provoquer une dégradation des habitats terrestres naturels, à la fois sur le site du projet et dans les voisinages (Mercier \& Bekhechi, 2003).

\section{Fragmentation des habitats}

La construction de chaussées, des routes d'accès et d'autres infrastructures à l'intérieur du site minier peut créer des barrières limitant les déplacements des espèces ou les empêchant d'accéder à des moyens vitaux (l'eau). On observe souvent un effet de lisière sur la verdure restante, puisque les routes ouvrent de nouveaux accès qui favorisent l'exploitation forestière, l'agriculture et la chasse à petite ou grande échelle (Mercier \& Bekhechi, 2003). 


\section{Perturbation des espèces}

Les perturbations provoquées par les activités minières (le bruit, les vibrations, la luminosité et la présence de personnes) peuvent arrêter les communications, les mouvements et les attitudes ou habitudes alimentaires et reproductifs des animaux (Kilosho et al., 2013 ; Mangambu et al., 2018). L'immigration de personnes peut provoquer des perturbations d'autant plus significatives ce que est énuméré ci-haut.

\section{Introduction d'espèces envahissantes}

Les espèces non indigènes sont une menace pour la biodiversité, car elles peuvent éliminer des espèces naturelles. Les espèces envahissantes peuvent être introduites par accident sur le site minier, par le biais d'équipements ou de matériaux en provenance d'autres régions. Si elles ne sont pas gérées de façon adéquate, elles peuvent provoquer la disparition d'espèces originaires du milieu (Mangambu et al., 2015). Ce risque est particulièrement grave sur les îles, où les espèces endémiques indigènes disposent de défenses naturelles limitées face aux espèces envahissantes.

\section{Morcellement de l'habitat}

Suite aux activités minières, le morcellement de l'habitat se produit lorsque de grandes portions de terres sont scindées en des parcelles de plus en plus petites, rendant difficile ou impossible la dispersion des espèces indigènes d'une parcelle à une autre entravant ainsi les routes migratoires naturelles. L'isolement peut conduire à un déclin des espèces locales ou des effets héréditaires comme la consanguinité. Les espèces qui nécessitent des parcelles importantes de forêts disparaissent (Mangambu et al., 2015).

\section{Perte de services écosystémiques}

La perte ou la dégradation des habitats et des espèces impactent également la subsistance et le bien-être des personnes. Les communautés dépendent des services, notamment pour l'eau potable, l'alimentation, le combustible et la plante médicinale (Mangambu et al., 2010).

\section{Impacts des projets miniers sur la qualité du sol Erosions et perturbation des activités agricoles}

L'exploitation minière peut contaminer le sol sur de vastes zones. Les activités agricoles proches d'un projet d'exploitation minière peuvent être particulièrement touchées. Selon une étude commanditée par l’Union européenne dans l'Ituri en RDC (Becker \& Vanclay, 2018), les opérations minières ont modifié le paysage environnant en exposant le sol qui était précédemment intact. L’érosion du sol exposé, les minerais extraits, les terrils et les matériaux fins dans les tas de déchets de roches peuvent entraîner des 
charges substantielles de sédiments dans les eaux de surface et les voies de drainage des eaux. Les déversements et fuites de matières dangereuses et les dépôts de poussières contaminées, fouettées par le vent peuvent conduire à la contamination du sol (Murphy et al., 1989)

\section{Contamination du sol}

Les risques sur la santé humaine et sur le sol appartiennent à deux catégories (Becker \& Vanclay, 2018): (1) sol contaminé provenant des poussières fouettés par le vent et (2) sol contaminé à partir de déversements de produits chimiques et des résidus. La poussière fugitive peut poser des problèmes environnementaux significatifs dans certaines mines. La toxicité de la poussière dépend de la proximité des récepteurs environnementaux et du type de minerai exploité. Des niveaux élevés d'arsenic, de plomb et de radionucléides dans la poussière fouettée par le vent constituent le plus grand risque.

La prolifération de pollutions aux métaux lourds présents dans le minerai d'or (arsenic et plomb en particulier), le rejet de cyanure dans la nature, la dissémination de mercure venant des mines artisanales, ou encore la pollution atmosphérique, présentent des dangers pour l'environnement et les espèces vivantes dont l'homme. Au Ghana, la pollution atmosphérique aux alentours des mines d'or industrielles a été associée à une incidence accrue de toux et à la fuite de la population autochtone (Aragón \& Rud, 2013).

Dans le bandeau de Liambe, le sol qui serait contaminé à partir des déversements de produits chimiques et des résidus sur les sites de la mine poserait un risque de contact direct, car ces matériaux sont utilisés abusivement comme matériaux de remblayage, pour la création de zones vertes ornementales ou encore comme suppléments de sol.

\section{Impacts des projets miniers sur la qualité du sol}

\section{Créations des érosions et de la perturbation des activités agricoles}

L'exploitation minière peut contaminer les sols sur de vastes zones. Les activités agricoles proches d'un projet d'exploitation minière peuvent être particulièrement touchées. Selon une étude commanditée par la Communauté européenne dans l'Ituri en RDC (Becker \& Vanclay, 2018) : les opérations minières ont modifié le paysage environnant en exposant des sols qui étaient précédemment intacts. L'érosion des sols exposés, les minerais extraits, les terrils et les matériaux fins dans les tas de déchets de roches peuvent entraîner des charges substantielles de sédiments dans les eaux de surface et les voies de drainage des eaux. En outre, les déversements et fuites de matières dangereuses et les dépôts de poussières contaminées fouettées par le vent peuvent conduire à la contamination du sol (Murphy et al., 1989). 


\section{Contamination du Sol}

Les risques sur la santé humaine et sur le milieu provenant des sols appartiennent généralement à deux catégories (Becker \& Vanclay, 2018): (1) sol contaminé provenant des poussières fouettés par le vent et (2) les sols contaminés à partir de déversements de produits chimiques et des résidus.

La poussière fugitive peut poser des problèmes environnementaux significatifs dans certaines mines. La toxicité inhérente de la poussière dépend de la proximité des récepteurs environnementaux et du type de minerai exploité. Des niveaux élevés d’arsenic, de plomb et de radionucléides dans la poussière fouettée par le vent constituent généralement le plus grand risque. La prolifération de pollutions aux métaux lourds naturellement présents dans le minerai d'or (arsenic et plomb en particulier), le rejet de cyanure dans la nature, la dissémination de mercure venant des mines artisanales, ou encore la pollution atmosphérique, présentent des dangers pour l'environnement et les espèces vivantes dont l'homme. Au Ghana par exemple, la pollution atmosphérique aux alentours des mines d’or industrielles a été associée à une incidence accrue de toux et à la fuite de la population autochtone (Aragón \& Rud, 2013).

Dans le bandeau de Liambe, le sol qui serait contaminé à partir des déversements de produits chimiques et des résidus sur les sites de la mine poserait un risque de contact direct, car ces matériaux sont utilisés abusivement comme matériaux de remblayage, pour la création de zones vertes ornementales ou encore comme suppléments de sol.

\section{Considérations sur les changements climatiques et les aléas naturels}

Les pays en développement surtout de l'Afrique font face à de nombreuses contraintes dues à la géologie du milieu, à sa localisation géographique, à l'histoire ou encore en cherchant un développement de son environnement économique...Toute étude d'impacts environnementaux et sociaux d'un projet minier qui a le potentiel pour modifier le bilan carbone global devrait inclure une évaluation de l'impact carbone du projet (Bonneuil et al., 2013). Les grands projets miniers ont le potentiel pour modifier le carbone global par la perte d'absorption de $\mathrm{CO}_{2}$ par les forêts et la végétation qui ont été abattues. Beaucoup de grands projets miniers sont proposés dans les zones fortement boisées des régions tropicales qui sont cruciales dans l'absorption de $\mathrm{CO}_{2}$ atmosphérique et dans le maintien d'une stabilité saine entre les projections et la consommation de $\mathrm{CO}_{2}$. Certains projets miniers conduisent à la destruction à long terme ou même permanente de forêts tropicales (Bryceson \& Jønsson, 2010 ; Lu, 2012).

Le programme d'EIE (Etude d'Impacts Environnementaux) pour les projets d'exploitation minière doit inclure une comptabilité minutieuse de la façon dont toute perturbation proposée des forêts tropicales modifierait le 
bilan carbone tel que proposent Bryceson et Jønsson (2010). L'EIES (Etude d'Impacts Environnementaux et Sociaux) doit aussi inclure une analyse du potentiel de perte du pays hôte de financement de consortiums internationaux qui sont et seront établis pour préserver les forêts tropicales (Busse et al., 2016 ; Dialga, 2017).

\section{Conclusion}

Les impacts environnementaux à devenir des grands projets miniers sont controversés et complexes. La question que pose la présente étude est de savoir comment les paysans résistent-ils contre ce projet, l'accaparement de leurs terres par les acteurs puissants. Dans la zone de Liambe et ses environs, qu'aucune des phases du cycle de mise en valeur des minéraux n'offre des possibilités économiques aux communautés riveraines. Soucieux de maximiser les gains d'un côté et de l'autre, préoccupé par le legs à la génération future, la relation de partenariat entre l'industrie minière et les autochtones sont devenues sources de mésententes énormes et souvent désavantageuses pour l'Etat Congolais. Il apparait que l'exploitation des ressources minières sur le lit de la rivière Aruwimi pourrait engendrer des calamités et des désastres sur les populations et sur l'environnement; ce qui rendrait le processus du développement plus difficile. Elle apporterait plutôt plus des problèmes que des solutions. Tout projet allant dans ce sens (d'exploitation minière) dans cette zone serait inopportun.

Au regard de la littérature scientifique parcourue et les réalités vécues sous d'autres cieux, la zone de Liambe et ses environs s'exposeraient aux conséquences diverses telles que les biotes associés par le biais de la suppression de la végétation ainsi que le sol de couverture, le déplacement de la faune, le dégagement de polluants et de bruit, etc. Les risques sanitaires et environnementaux induits pendant l'exploitation, après les fermées des mines sont d'émanation de gaz dangereux, pollutions de l'eau, de l'air et des sols, mouvements de terrain et inondations. Cela risquerait d'ouvrir la boîte aux pandores si ces érosions touchaient les tourbières de la région...et libérer par conséquent, le $\mathrm{CO}_{2}$ emprisonné !

Les concessions minérales dans les carrés en dehors de la surface d'eau se trouvant sur le territoire des peuples indigènes subiraient menaces d'acculturation, voire de disparition. Et aussi des pertes économiques pour les populations locales (mort du bétail et dégâts pour les cultures, diminution de la productivité des sols à cause de la pollution), etc. Hormis ces aspects soulevés dans ce papier, nous demandons aux autres acteurs et autres chercheurs qui aborderont une telle thématique, d'intégrer d'une manière adéquate aussi des aspects socioculturels et économiques dans les évaluations environnementales pour mieux prévenir et gérer les risques et leurs conséquences. 
Ce projet peut créer des richesses à la firme chinoise, mais également provoquer des perturbations environnementales considérables, difficiles à corriger. Si les impacts sur la population et le milieu humain sont ignorés ou sous-estimés dans les EIES (pas de mesures) et PGES (Plan de Gestion Environnementale et Sociale) cela entraine des lourdes conséquences d'ordre social et économique.

Tous ceux-ci montrent clairement que l'exploitation des ressources minières sur le lit de la rivière Aruwimi pourrait engendrer des calamités et des désastres sur les populations et sur l'environnement ; ce qui rendrait le processus développement plus difficile. Elle apporterait plutôt plus des problèmes que des solutions. La communauté autochtone n'aura pas de moyens de recours à ses propres outils pour réparer les différents dégâts. Les conflits opposant les communautés locales à société minière ont reçu ainsi un important écho médiatique et nous des ONG environnementalistes avons développé des campagnes visant à attirer l'attention de l'opinion publique sur les nuisances environnementales et sociales de ce projet minier.

\section{Remerciements}

Les Chercheurs impliqués dans cette étude remercient de tout cœur toutes les personnes qui ont accepté volontiers de collaborer avec eux lors de l'enquête sur le terrain. Ils remercient également les Agents de l'État pour leur noble contribution à l'élaboration du rapport. Leur remerciement s'adresse enfin, à la population qui a consacré son temps à l'enquête. Ils espèrent que cette recherche contribuera à une meilleure coopération entre toutes les parties prenantes. Le premier et le deuxième auteur rendent hommage au Feu Professeur Ekele Mbenga (troisième auteur) qui a sponsorisé une grande partie de cette recherche. Malheureusement, il fut mort dans des conditions encore non élucidées, que son âme repose en paix.

\section{References:}

1. Abadie, J.C. (2008). La nature ordinaire face aux pressions humaines : le cas des plantes communes. Méthodes de suivis et évaluation de l'impact des activités humaines, Paris, Musée national d'histoire naturelle, $206 \mathrm{p}$

2. Acar, E. F. \& Sun, L. (2013). A Generalized Kruskal-Wallis Test Incorporating Group Uncertaintywith Application to Genetic Association Studies, Biom, 69, pp. 427-435.

3. Actuar en Cristianos Informados - RDC (2009). Exploitation minières au Katanga : un atout pour le développement ou une colonisation économique ? Lubumbashi/RDC, $30 \mathrm{p}$.

4. Andre, P., Delisle, C.-E. \& Reveret, J-P. (2010). L'évaluation des impacts sur l'environnement. Processus, acteurs et pratique pour un 
développement durable. Montréal: Presses internationales Polytechnique, 3e édition.

5. Anonyme (2016). Rapport de Territoire de Basoko 2016 : Données du Bureau d'Etat civil de Basoko de l'exercice 2015-2016, 42 p.

6. Aragón, F. M. \& Rud, J.P. (2013). Evidence from a Peruvian Gold Mine. American Economic Journal: Economic Policy, 5 (2), pp. 1-25

7. Ashanti Goldfields Kilo (2015). Ce Résumé d'Etude d'Impact Environnemental et du Plan de Gestion Environnemental du projet minier Mongbwalu, Kinshasa/RDC 46 p, http://congomines.org/reports/788-resume-de-l-etude-d-impactenvironnemental-et-du-plan-de-gestion-environnemental-du-projetmongbwalu

8. Asimbo, B.N. \& Mangambu, M.J. (2021). Vision pragmatique des impacts socio - économiques de l'exploitation minière semi industrielle sur la rivière Aruwimi : un mauvais paradigme sur les cours d'eaux du Bassin du Congo (Territoire de Basoko, RD Congo). Soumission manuscrit_EJS_Sciences Sociales 0674/21

9. ATSDR (Agency for Toxic Substances and Disease Registry) (2011). Toxicological profile for cyanide. http://www.atsdr.cdc.gov/toxprofiles/tp.asp?id=72\&tid=19

10. Bebbington, A., Hinojosa, L., Humphreys Bebbington, D., Burneo, ML. \& Warnaars, X. (2008). Contention and Ambiguity: Mining and the Possibilities of Development, Brooks World Poverty Institute Working Paper No. 57, Manchester: Brooks World Poverty Institute.

11. Becker, H. \& Vanclay, F. (2018). The International Handbook of Social Impact Assessment: Concept and Methodological Advances. Edward Algar. Cheltenham UK. pp. 74-91.

12. Berzins, R., Claro, F., Akpona, A. H. \& Gambari Imorou, S. (2007). Conservation du guépard etdéveloppement durable dans les aires protégées du nord Bénin. Mission d'enquête auprès des villageoiset des agents d'aires protégées (16/12/2005 au 26/02/2006). Paris, Société Zoologique de Paris, 57 p.

13. Bonan, G.B. (2008). Forests and Climate Change: Forcings, Feedbacks, and theClimate Benefits of Forests. Science, vol. 320 p. www.sciencemag.org

14. Bonneuil, C., Pessis, C., Topcçu, S. et al. (2013). Une autre histoire des "Trente Glorieuses" : Modernisation, contestations et pollutions dans la France d'après-guerre. Paris, La Découverte, 132 p.

15. Breshears, D.D., López-Hoffman, L. \& Graumlich, L. J. (2019). When Ecosystem Services Crash: Preparing for Big, Fast, Patchy Climate Change. AMBIO, Journal of Environment and Society, 40 pp. 256263.https://link.springer.com/article/10.1007\%2Fs13280-010-0106-4 
16. Bryceson, D.F. \& Jønsson, J.B. (2010). Gold digging careers in rural east Africa: Small-scale miners' livelihood choices. World Development, 38(3), pp. 379-392.

17. Busse, M. \& Gröning, S. (2013). The resource curse revisited: governance and natural resources. Public Choice, 154(1-2):1-20. Hinton J, Veiga, MM. \& Beinhoff, C. (2003). Women, mercury and artisanal gold mining: risk communication and mitigation. Journal de Physique IV. 107, pp. 617-620.

18. Busse, M., Erdogan, C. \& Mühlen, H. (2016). China's Impact on Africa - The Role of Trade, FDI and Aid. Kyklos, 69(2), pp. 228-262.

19. Coleman J., Guven, B., Johnson, L. \& Sachs, L. (2018). What do we mean by investment facilitation? Columbia Center on Sustainable. Investment.http://ccsi.columbia.edu/2018/02/22/what-do-we-meanby-investment-facilitation/Documents

20. Coulibaly, G.M.M. (2012). Evaluation environnementale et analyse des risques dans l'exploitation minière : conséquences du non-respect des obligations environnementales.

https://www.sifee.org/static/uploaded/Files/ressources/actes-descolloques/lome/session-3-3/COULIBALY_DIAPO.pdf

21. Cyuzuzo, L. (2020). L'exploitation minière à l'Est de la République Démocratique du Congo (RDC): Réflexions sur les enjeux économiques, politiques et environnementaux d'une enquête ethnographique. Mémoire de Master I de l’École des Hautes Études en Sciences Sociales, $53 \mathrm{p}$

22. De Saint Moulin, L. \& Kalombo, J-L.T. (2005). Atlas de l'organisation administrative de la République Démocratique du Congo. Centre d'études pour l'action sociale (Kinshasa, Congo), 2e Edition revue et amplifiée

23. Décret $n^{\circ}$ 038/2003 du 26 mars 2003 portant Règlement minier tel que modifié et complété par le Décret n 18/024 du 08 juin 2018

24. Dialga, I. (2017). Un développement durable fondé sur l'exploitation minière est-il envisageable ? Élaboration d'un Indice de Soutenabilité des Pays Miniers appliqué au Burkina Faso et au Niger. Thèse de Doctorant, Université Bretagne Loire

25. Dmoergue, C. \& Mpoyi, M.A. (2012). La gestion des ressources naturelles pour une croissance durable. In Herderschee, J., Mukoko Samba, D. \& Tshimenga Tshibangu, M. (éd.), Résilience d'un géant africain : accélerer la croissance et promouvoir l'emploi en République démocratique du Congo. Études sectorielles, Kinshasa : Médiaspaul, 2. pp. 99-183.

26. Ekele, M. \& Mangambu, M. (2020). Feuilleton sur l'exploitation des minerais par la société Xiang Jiang Mining sarl, une entreprise 
chinoise a Basoko (Province de la Tshopo, RD Congo). Edition les Ekeles, Kinshasa, pp. 1- 14

27. Gavard-Perret, M.L., Gotteland, D., Haon, C. \& Jolibert, A. (2011). Méthodologie de la recherche. Réussir son mémoire ou sa thèse en sciences de gestion, Paris, Pearson, 383 p.

28. Glasson, J. \& Therivel, R. (2013). Introduction to environmental impact assessment (4th ed.). London, Routledge, $242 \mathrm{p}$.

29. Grzybowski, A. (2012). Guide pratique pour la prévention et la gestion des conflits liés à la terre et aux ressources naturelles : industries extractives et conflits. Le Groupe interagences des Nations Unies pour les actions préventives, $52 \mathrm{p}$

30. Hanson, T., Brooks, M., da Fonseca, G.A.B., Hoffman, M., Lamoreaux, J.F., Machlis, G.E., Mittermeier, C.G., Mittermeier, R.A. \& Pilgrim, J.D. (2009). 'Warfare in Biodiversity Hotspots', Conservation Biology, 23(3), 2009, pp. 578-587.

31. Homer-Dixon, T. (1994). 'Environmental Scarcities and Violent Conflict: Evidence from Cases’, International Security, 19(1), pp. 540.

32. Howell, C. (2004). Is There a Third Way for Industrial Relations? An International Journal of Employment Reletions, 42 (1), pp. 1-22. https://doi.org/10.1111/j.1467-8543.2004.00302.x

33. Hynes, S., Ghermandi, A., Norton, D. \& Williams, H. (2018). Marine recreational ecosystem service value estimation: A meta-analysis with cultural considerations. Ecosystem Services, vol. 31, p. 410-419.

34. IFDD (Institut de la Francophonie pour le Développement Durable) (2019). Francophonie et développement durable : Innovations et bonnes pratiques.Marquis Imprimeur, Canada, 147 p. ISBN: 978-289481-292-1. www.ifdd.francophonie.org

35. Justice et Paix de la Fédération Wallonie-Bruxelles (2019). Code minier en RD Congo : les enjeux de la Réforme. www.justicepaix.be

36. Kamundala, G. (2012). Exploitation minière Industrielle et artisanale au Sud Kivu: Possibilités d'une cohabitation pacifique? Coopération Universitaire Flamande (VLIR-UOS), Université d'Anvers, 23 p

37. Keenan, K., de Echave, J. \& Traynor, K. (2002). Mining and communities: Poverty amidst. Wealth. International Conference on Natural Assets, Conference Paper Series No.3. 25.

38. Kemp, D. (2009). Community Relations in the Global Mining Industry: Exploring the Internal Dimensions of Externally Orientated Work, Corporate Social Responsibility and Environmental Management. Ministerial Council on Mineral and Petroleum Resources (MCMPR). 2005. Principles of Engagement with Communities and Stakeholders. Canberra. 
39. Kilosho, J., Mukasa, A.N. \& Kamundala, G. (2013). Traçabilité des produits miniers dans des zones de conflits au Sud Kivu, In Marrysse,S. \& Omasombo, J.(éd.), Collection Cahiers africains, Conjonctures congolaises 2012 nº $^{\circ}$. Paris, l’Harmattan, pp. 11514224.

40. Lanmafankpotin, G., André, P., Samoura, K., Côté, L., Beaudet, R., \& Bernier, M. (2013). La participation publique dans l'évaluation environnementale en Afrique francophone. Québec, Institut de la Francophonie pour le développement durable. Points de repère No. 23. ttps://www.sifee.org/static/uploaded/Files/publications/membres/Part _publique_EE_Afrique_franco.pdf

41. Lara-Pulido, J. A., Guevara-Sangines, A. \& Martelo, C.A. (2018). A meta-analysis of economic valuation of eco-system services in Mexico. Ecosystem Services, 31, pp. 126-141

42. Loi $n^{\circ} 007 / 2002$ du 11 Juillet 2002 portant Code minier telle que modifiée et complétée par la Loi n¹8/001 du 09 Mars 2018.

43. Lu, J.L. (2012). Occupational health and safety in small scale mining: focus on women workers in the Philippines. Journal of International Women's Studies. 13(3) pp. 103-113.

44. Mangambu, M. (2021). Exploitation minière semi -industrielle dans la rivière Aruwimi (Territoire de Basoko en RD CONGO) : Analyse d'impact socio-environnemental au regard des exigences du développement durable des communautés locales. Livre sous presse dans les éditions Arco/Belgique.

45. Mangambu, M., Ntahobavuka, H., Basinyize, B., Lokumu, I. \& Robbrecht, E. (2018). Pteridaie: indicator of the vegetation succession and dynamics in the forests Chablis and Edges in Kahuzi-Biega National Park (Eastern D. R. Congo, Albertine Rift). International Journal of Biological Research, 6 (1), pp. 5-13. http://dx.doi.org/10.14419/ijbr.v6i1.9241

46. Mangambu, M., Habiyaremye, F., Lina, A. \& Ntahobavuka, H. (2010). L'importance du groupement à Cyathea manniana Hook. dans la biodiversité du Parc National de Kahuzi -Biega, R. D. Congo. GeoEco-Trop, 34 (1/2) pp. 45-63.

47. Mangambu, M., Aluma, K., van Diggelen, R., Rugenda-Banga, R., Mushangalusa, K., Chibembe, S., Ntahobavuka, H., Nishuli, B. \& Robbrecht, E. (2015). Etudes ethnobotanique et ethnolinguistique des ressources forestières ligneuses utilisées par la population du couloir écologique du parc national de Kahuzi-Biega (R D. Congo). European Scientific Journal, 11 (15), pp. 135-162. http://dx.doi.org/10.19044/esj.2014.v.183 19 
48. Marysse, S. \& Tshimanga, C. (2013). Les trous noirs de la rente minière en RDC, In. S. Marysse et J. Omasombo (eds), Conjonctures congolaises 2013 : Percée sécuritaire, flottements politiques et essor économique, Paris, L'Harmattan.26.

49. Matthysen, K., Hilgert, F., Schouten, P. \& Mabolia, A. (2011). Une analyse détaillée du secteur de l'or en Province Orientale. GIZ- PIS, Anvers/Belgique, $211 \mathrm{p}$.

50. Mazalto, M. (2009). "Governance, human rights and mining in the Democratic Republic of Congo ». In B. Campbell (éd.), Mining in Africa. Regulation and Development. London and New York : Pluto Press, pp. 187-242.

51. Mercier, J.R. \& Bekhechi, M.A. (2003). L'évaluation environnementale: un outil majeur du développement durable. Revue africaine de gestion et d'évaluation environnementales 5, pp. 102-139. http://www.ajeam-ragee.org/vol5p102t139.htm

52. Moreau, A., Dedianne, M.-C., Letrilliart, L., Le Goaziou, M-F., Labarère, J. \& Terra, J.L. (2004). S'approprier la méthode du focus group, La revue du praticien-médecine générale, 645, 18 pp. 382-384

53. Mugangu, S. (2008). La crise foncière à l'Est de la RDC, In : Marysse S., F. Reyntjens et Vandeginste, S. (eds.), L'Afrique des Grands Lacs. Annuaire 2007-2008, Paris, L'Harmattan, 385-414

54. Murphy, C.A., Coover, D.O. \& Steven, V. (1989). Educational and Psychological Measurement 49(4) pp.893-899. https://doi.org/10.1177/001316448904900412

55. Nogier, J-F. (2008). Ergonomie du logiciel et design web : Le manuel des interfaces utilisateur, 4e édition, Dunod , 212p. ISBN : 9782100515721

56. Oberiuele, L. (2011). Rapport de recherche sur la production et la commercialisation d'or dans la ville d'Isiro. Rapport de recherche commandé par IPIS/International Alert, $132 \mathrm{p}$.

57. OMS (1948). Glossaire de la promotion de la santé, Genève/Suisse, $27 \mathrm{p}$

58. Otto, J.M. (2009). Global trends in mines reclamation and closure regulation. In J. Richard (ed.) Mining, Society, and a Sustainable World (251-288), Berlin: Springer.

59. PNUD (2012). A practical guide: reducing mercury use in artisanal and small-scale gold mining. Geneva: United Nations Environment Programme, 176 p. http://web.unep.org/fr

60. PNUD (2016). Évaluation environnementale de la pollution au mercure dans l'est de la République Démocratique du Congo. Rapport Technique pour le projet « or juste » du partenariat Afrique-canada, Nairobi/Kenya, 76 p. http://web.unep.org/fr 
61. PUND (2016). Évaluation environnementale de la pollution au mercure dans l'est de la République Démocratique du Congo : BUTUZI au SUD KIVU et SOME en ITURI. Rapport technique pour le projet « or juste » du partenariat Afrique - canada. Programme des Nations Unies pour l'Environnement 2, Nairobi, KENYA, $76 \mathrm{p}$ http://web.unep.org/fr

62. SAESSCAM (2019). Service d'assistance et d'encadrement du secteur minier de type artisanal, Antenne de Kisangani, Rapport mensuel juin 2019, inédit.

63. Schröter, D., Wolfgang, C., Leemans, R. I., Prentice, C., Miguel, B., Nigel, W., Bondeau, A., Bugmann, H., Timothy, R., Carlos, A., de la Vega-Leinert, C.A., Markus, E., Ewert, F., Glendining, M J. I., House, S., Kankaanpää, R.J., Klein, T., Lavorel, S., Lindner, M., Metzger, M.J., Meyer, J., Mitchell, T.D., Reginster, I., Rounsevell, M., Sabaté, S., Sitch, B., Smith, J., Smith, P., Smith, M.T., Sykes, K.W., Thonicke, T., Tuck, G., Sönke, Z.B. \& Zierl, Z. (2005). Ecosystem Service Supply and Vulnerability to Global Change in Europe. Scienceexpess. http://dx.doi.org/110.1126/science.1115233

64. Stratos, I. (2011). Climate Change and Acid Rock Drainage - Risks for the Canadian Mining Sector. MEND, Ottawa, Canada. MEND Report 1.61.7. http://mend-nedem.org/wp-content/uploads/2013/01/1.61.7.pdf

65. Thomas Lassourd (2018). La fiscalité du nouveau code minier de la République Démocratique du Congo, Natural Resource Governance Institute, Analyse $32 \mathrm{P}$, www.resourcegovernance.org.

66. Touré, E.A. (2010). Réflexion épistémologique sur l'usage des focus groups : fondements scientifiques et problèmes de scientificité. Dans Guillemette F., Luckerhoff J. et Baribeau C.,Entretiens de groupe : concepts, usages et ancrages, Recherches qualitatives, 29 (1), pp 5-27.

67. Vaessen, V. \& Brentführer, R. (2015). Les risques liés à l'eau souterraine : intégration de la gestion des eaux souterraines dans les organismes de bassin transfrontaliers en Afrique. Hannover, Allemagne, $20 \mathrm{p}$.

68. Wakenge, C. (2019). Stade Coltan: extraction minière artisanale, réformes et changement social à l'Est de la RDC. Le harmattan, Collection Etudes Africaines, 248 p. 